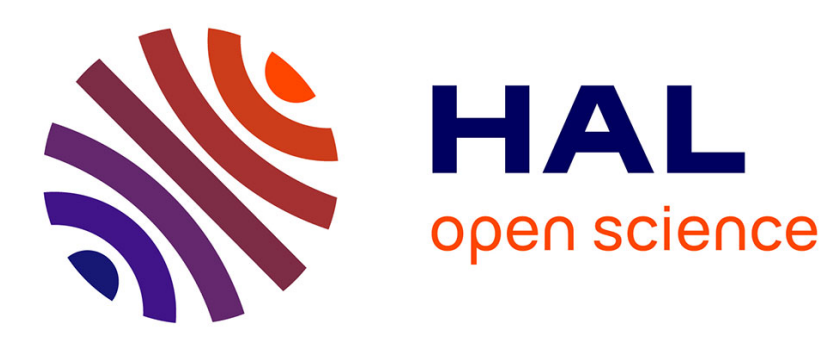

\title{
Time analysis of the three characteristic behaviours of dual-porosity media. I: fluid flow and solute transport Pascale Royer, Claude Boutin
}

\section{To cite this version:}

Pascale Royer, Claude Boutin. Time analysis of the three characteristic behaviours of dual-porosity media. I: fluid flow and solute transport. Transport in Porous Media, 2012, 95 (3), pp.603-626. $10.1007 / \mathrm{s} 11242-012-0065-2$. hal-00786967

\section{HAL Id: hal-00786967 https://hal.science/hal-00786967}

Submitted on 11 Feb 2013

HAL is a multi-disciplinary open access archive for the deposit and dissemination of scientific research documents, whether they are published or not. The documents may come from teaching and research institutions in France or abroad, or from public or private research centers.
L'archive ouverte pluridisciplinaire HAL, est destinée au dépôt et à la diffusion de documents scientifiques de niveau recherche, publiés ou non, émanant des établissements d'enseignement et de recherche français ou étrangers, des laboratoires publics ou privés. 
manuscript No.

(will be inserted by the editor)

\title{
Time analysis of the three characteristic behaviours of dual-porosity media. I: fluid flow and solute transport
}

\author{
P. Royer · C. Boutin
}

the date of receipt and acceptance should be inserted later

\begin{abstract}
Homogenisation of consolidation and compressible fluid flow in dual-porosity media has highlighted the existence of three characteristic macroscopic behaviours. These three behaviours are, namely, a dual-porosity description which includes memory effects, a single-porosity description with which the micro-porosity is simply ignored, and an intermediate behaviour which we refer as behaviour with reservoir effect. With this latter, the whole dual-porosity medium is represented by an equivalent single-porosity medium. In contrast with a single-porosity behaviour, the porosity of the entire dual-porosity medium is accounted for. During solute transport in dual-porosity media, while memory effects are most often experimentally observed, the homogenised model obtained for the most general values of the involved parameters leads to a model with reservoir effect. Therefore, the observed memory effects are not reproduced by this model and a clear interpretation of the origins of these effects remains an unresolved issue. The work is presented in two complementary articles. The objective of the present paper is, firstly, to determine a physical interpretation of the existence of the three characteristic behaviours of dual-porosity media. This is performed by exploring the homogenised models and their domains of validity for the analogy of heat conduction in a dual-conductivity composite. This leads to the original result that consists to relate each type of behaviour to a specific relationship between two characteristic times. This is then used for interpreting the results obtained for compressible flow in dual-porosity media. Finally, it allows to elucidate the conditions under which memory effects may occur during solute transport in dual-porosity media.
\end{abstract}

Keywords Dual-conductivity $\cdot$ Dual-porosity $\cdot$ Fluid flow $\cdot$ Solute Transport $\cdot$ Homogenisation of multiple scale asymptotic expansions

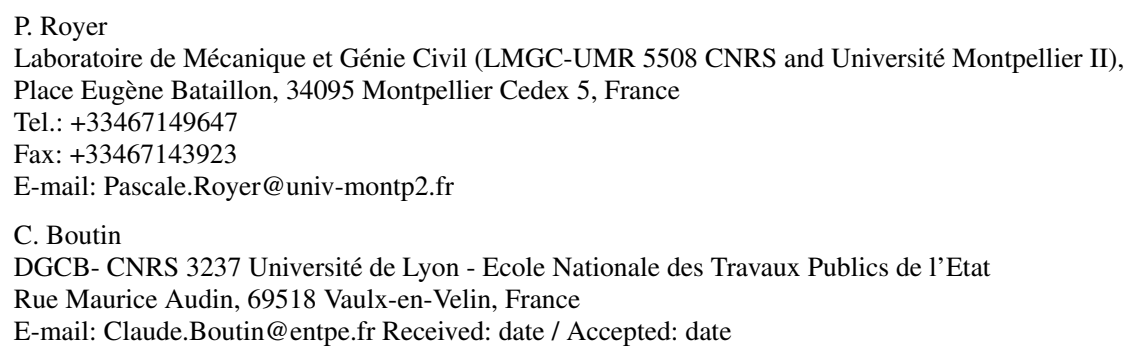




\section{Introduction}

The focus of the present study is on the fundamentals of fluid flow, consolidation and solute transport in dual-porosity media, i.e. in media that consist of two interacting porous systems of distinctly different fluid transfer properties. When considering a transient phenomenon in such structures, memory effects may occur. Predicting and modelling these effects constitute the key issues in studying any phenomenon in dual-porosity media and have been the subject of a wide bulk of research since the introduction of dual-porosity concept and first models (Barenblatt et al. 1960; Barenblatt 1963; Warren and Root 1963). Intended for modelling fluid flow in fractured porous media, these models are two-equation models: one equation describes fluid flow in the network of fractures while the other one governs the flow within the porous matrix. The exchange of fluid between both porous structures is described by means of a coupling term which is proportional to the pressure difference. The use of twoequation models has then been introduced for describing solute transport (Coats and Smith 1964; Bibby 1981; Huyakorn et al. 1983) and consolidation (Wilson and Aifantis 1982; Khaled et al. 1984; Beskos and Aifantis 1986; Elsworth and Bai 1992; Bai et al. 1993; Berryman and Wang 1995) in dual-porosity media.

The first results by using the homogenisation method of multiple scale asymptotic expansions were obtained with a two-scale approach by Auriault (1983) for the analogy of heat conduction in dual-conductivity media and in (Arbogast 1989) and (Arbogast et al. 1990) for compressible fluid flow in dual-porosity media. "Dual-porosity" models, i.e. models with memory effects are thus obtained which are non-local in time and which do not reduce to two-equation models of Barenblatt's type (Auriault and Royer 1993; Hornung and Showalter 1990). The computational issues for solving these homogenised models are described in (Arbogast 1997).

Dual-porosity media are characterised by the existence of three characteristic lengths of observation. In the sequel, we consider a dual-porosity medium made of meso-pores surrounded by blocks of a micro-porous matrix. The three scales are, namely: the micropore scale, the mesoscopic (matrix/mesopore) scale and the macroscopic scale. Application of a three-scale homogenisation procedure has led to the identification of three characteristic behaviours for quasi-static consolidation and highly compressible fluid flow in dualporosity media (Auriault and Boutin 1992), (Auriault and Boutin 1993), (Royer and Auriault 1994),(Royer et al. 1996), (Boutin et al. 1998). These three behaviours include a dual and a single porosity description, and an intermediate behaviour which we refer as behaviour with reservoir effect. With this latter, the whole dual-porosity medium is represented by an equivalent single-porosity medium. In contrast with a single-porosity behaviour, the porosity of the entire dual-porosity medium is accounted for. It was shown that the three models are obtained for three distinct relationships between the scale ratios $\alpha=l_{\mathrm{m}} / l_{\mathrm{p}}$ and $\varepsilon=l_{\mathrm{p}} / \mathrm{L}$, where $l_{\mathrm{m}}, l_{\mathrm{p}}$ and $\mathrm{L}$ denote the micro-pore size, the mesopore size and the macroscopic size, respectively. For passive solute transport in dual-porosity media, while many experimental works report that memory effects occur when the transport mechanism is purely diffusive within the micro-porosity and dispersive in the mesoporosity, the corresponding model obtained by homogeneisation is a model with reservoir effect (Royer et al. 2002). This means that the derived model doesn't reproduce the experimentally observed dual-porosity effects and might suggest that, in most cases, memory effects during solute transport in dual-porosity media may not be represented by a continuum macroscopic description. Anyhow, a question which arises naturally from this result inquires about the origins of memory effects during solute transport in dual-porosity media. 
The present work is aimed towards providing a global interpretation of the results obtained in former works related to various phenomena in dual-porosity media (Auriault and Boutin 1992), (Auriault and Boutin 1993), (Royer and Auriault 1994),(Royer et al. 1996), (Boutin et al. 1998), (Royer et al. 2002). The objective is two-fold:

1. to explain and interpret the existence of three characteristic behaviours of dual-porosity media;

2. to determine the conditions under which memory effects occur during solute transport in dual-porosity media.

The work is presented in two separated papers. In the present paper, we firstly briefly introduce the homogenisation method that has been used for deriving the models and their domains of validity which will be presented and commented in the sequel. Next, we consider the analogy of heat conduction in a dual-conductivity composite medium. We present the associated homogenised models together with their domains of validity and we show how these domains of validity can be translated into orders of magnitude of a characteristic time ratio. Then, we focus on the use of this fundamental result for interpretating the results obtained in former works for compressible fluid flow and solute transport in dualporosity media. This allows to provide a clear interpretation of the existence of the three characteristic behaviours for compressible fluid flow in dual-porosity media. Translating this fundamental rule for solute transport in dual-porosity shows that, due to the existence of two characteristic times in the phenomenon of transport, the origin of memory effects is distinct than that observed for compressible flow. In particular, it is shown that under specific conditions, memory effects may occur which can't be described in the framework of an equivalent continuum macroscopic description. Two homogenised models are furthermore presented together with their domains of validity: a model with reservoir effect and a model with memory effects.

In the second paper (Royer and Boutin 2012), we examine the behaviours obtained for consolidation in dual-porosity media and apply the aforementioned result in terms of characteristic times for interpretating their domains of validity. In both papers, besides the interpretation of the macroscopic behaviours, several aspects presented in the initial works are corrected or clarified.

\section{Homogenisation method}

The homogenisation method for periodic structures, also called method of multiple scale asymptotic expansions was introduced by Sanchez-Palencia (1980) and Bensoussan et al. (1978). This method is a powerful tool for determining the macroscopic behaviour of a heterogeneous medium subjected to the action of a given phenomenon, by starting from the governing equations at the heterogeneity scale. As any continuum modelling approach, the method is based upon the fundamental assumption of separation of scales. The second assumption made is the periodiciy of the medium which is the simplest and the most efficient mathematical way to formulate the notion of elementary representative volume. The advantage of the method is that, thanks to the periodicity, no preliminary assumption is required on the form of the macroscopic models. It should also be underlined that the periodicity has no impact on the form of the macroscopic models.

Consider a periodic heterogeneous medium of large dimensions, submitted to a phenomenon fluctuating according to the characteristic macroscopic size L. Let further denote 
the periodic cell by $\Omega$ and its characteristic length by $l$. The fundamental condition of separation of scales is expressed by: $\varepsilon=l / \mathrm{L} \ll 1$. To describe the variations at the distinctly different lengths $\mathrm{L}$ and $l$, two space variables are introduced, $\mathbf{x}$ for the macroscopic variations, $\mathbf{y}$ for the microscopic variations, $\mathbf{x}$ and $\mathbf{y}$ being related by the scale ratio $\mathbf{y}=\varepsilon^{-1} \mathbf{x}$. Hence, the spatial derivative is expressed as: $\varepsilon^{-1} \partial_{y}+\partial_{x}$. The use of the small parameter $\varepsilon$ suggests to look for physical unknowns $q$ in the form of asymptotic expansions in powers of $\varepsilon$. The assumption of material periodicity and the condition of separation of scales entail that the physical quantities be $\Omega$-periodic with respect to variable $\mathbf{y}$ :

$q(\mathbf{x}, \mathbf{y})=\sum_{0}^{\infty} \varepsilon^{i} q^{i}(\mathbf{x}, \mathbf{y})$ with $q^{i}(\mathbf{x}, \mathbf{y}) \Omega$-periodic in $\mathbf{y}$.

A key point of this asymptotic approach is that the small parameter $\varepsilon$ is also used for characterising the physics of the phenomenon being considered. This clearly appears in the methodology introduced by Auriault (1991). This latter consists in placing the local governing equations into a dimensionless form, which gives rise to dimensionless parameters in the writing of the dimensionless equations. The orders of magnitude of these dimensionless parameters are estimated in power of scale ratio $\varepsilon$, accordingly to the situation under consideration. Introducing these estimates into the two-scale differential set - where the spatial gradient reads $\varepsilon^{-1} \nabla_{y}+\partial_{x} \nabla_{x}$ and $\mathbf{y}$ and $\mathbf{x}$ are from now on treated as two independent space variables - provides the rescaled equations on which the homogenisation process is performed. Identifying the terms of the same power in $\varepsilon$, and solving the boundary value problems obtained in series yield the first non trivial balance equation. This defines the homogenised model - restricted to the leading order and depending on the considered rescaling - as a "limit model" obtained for $\varepsilon \rightarrow 0$.

The remaining question is to identify the relevancy of these idealised models when applied to real materials of finite sizes (microscopic and macroscopic). The present work is aimed at investigating this point for heat transfer in dual conductivity composites and mass or solute transfer in dual-porosity media. The argument (Boutin and Auriault 1990) is based on the duality between the mathematical $\varepsilon$ used in the asymptotic process $(\varepsilon \rightarrow 0)$, and the actual physical scale ratio $\varepsilon_{r}$ which remains of finite value. In particular, the fact that $\varepsilon_{r}$ has a finite value is used to determine the relevant rescaling of the equations governing the physics of the phenomenon under consideration. Hence, the homogenised description associated to this rescaling is consistent with the actual physics occurring in the real media. This assertion relies on the convergence of the homogenisation process, enabling to state that the idealised description reached at the limit $(\varepsilon \rightarrow 0)$ is close to the description in the vicinity of the limit $\left(\varepsilon_{r} \ll 1\right)$ that would correspond to the real case.

When distinct orders of magnitude of dimensionless parameters lead to distinct macroscopic models - as in the present work - it turns out that the domain of validity of a given macroscopic model is expressed by the corresponding orders of magnitude of the dimensionless parameters. In this paper, we show how the physical scale ratio $\varepsilon_{r}$ enables to translate these dimensionless domains of validity into simple physical arguments.

\section{Macroscopic models for thermal conduction in dual-conductivity media}

\subsection{Introduction}

Heat conduction in a dual-conductivity medium, i.e. in a binary composite medium with a conductivity contrast constitutes a convenient analogy for the mathematical modelling of 
transport phenomena in dual-porosity media. In this section, we firstly present the four distinct transient macroscopic models obtained by homogenisation together with their domains of validity. Two of these models have been obtained in (Auriault 1983) and the two other models can easily be deduced from the results of this work. Next, we focus on the interpretation of the domains of validity of the models. Finally, we estimate the order of magnitude of the local flux ratio, as it is an important parameter for homogenising transient fluid flow in dual-porosity media.

\subsection{Derivation of the macroscopic models}

\subsubsection{Medium description}

We consider a periodic composite medium of period $\Omega$ (Fig. 1) and we assume that the dimension of the period, $l$, is small compared to the characteristic macroscopic length L: $\varepsilon=$ $l / \mathrm{L} \ll 1$. The period is composed of two constituents, which we distinguish by subscripts 1 and 2 , and which occupy domains $\Omega_{1}$ and $\Omega_{2}$, respectively. The boundary between both constituents is denoted by $\Gamma$. We introduce the following averages:

$$
<\cdot>_{\Omega}=<\cdot>_{\Omega}^{\Omega_{1}}+<\cdot>_{\Omega}^{\Omega_{2}}, \quad \text { where }<\cdot>_{\Omega}^{\Omega_{\gamma}}=\frac{1}{|\Omega|} \int_{\Omega_{\gamma}} \cdot d \Omega
$$

We assume that constituent 1 is connected and more conductive than constituent 2 , and

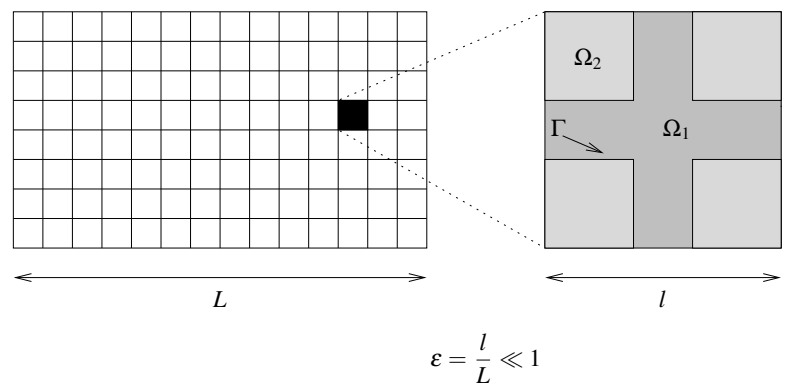

Fig. 1 Periodic composite medium.

that both thermal capacities are of same order of magnitude, which in the framework of homogenisation is expressed by: $\lambda_{2} / \lambda_{1} \leq \mathscr{O}\left(\varepsilon^{0}\right)$ and $\rho_{2} c_{2} / \rho_{1} c_{1}=\mathscr{O}\left(\varepsilon^{0}\right)$. In the sequel, quantities indexed by c denote characteristic values, e.g. $\lambda_{1}=\mathscr{O}\left(\lambda_{1_{\mathrm{c}}}\right)$, while stared parameters stand for the dimensionless ratio of real to characteristic values e.g.: $\lambda_{1}^{*}=\lambda_{1} / \lambda_{1_{\mathrm{c}}}$.

\subsubsection{Dimensionless local governing equations}

Within the periodic cell, heat conduction is described by the following set of equations, where $T_{\gamma}$ and $\boldsymbol{q}_{\gamma}$ stand for the temperature field and the heat flux in constituent $\gamma(\gamma=1,2)$, 
respectively:

$$
\left\{\begin{array}{l}
\boldsymbol{\nabla} .\left(\boldsymbol{q}_{\gamma}\right)+\rho_{\gamma} c_{\gamma} \frac{\partial T_{\gamma}}{\partial t}=0 \quad \text { in } \Omega_{\gamma},(\gamma=1,2), \\
\boldsymbol{q}_{\gamma}=-\tilde{\lambda}_{\gamma} \boldsymbol{\nabla}\left(T_{\gamma}\right) \quad \text { in } \Omega_{\gamma},(\gamma=1,2), \\
T_{1}=T_{2} \quad \text { on } \Gamma, \\
\left(\tilde{\lambda}_{1} \boldsymbol{\nabla} T_{1}\right) \cdot \mathbf{n}=\left(\tilde{\lambda}_{2} \nabla T_{2}\right) \cdot \mathbf{n} \quad \text { on } \Gamma .
\end{array}\right.
$$

Choosing the macroscopic length $\mathrm{L}$ and a characteristic time $t_{\mathrm{c}}$ as the reference length and time, respectively, the above set of equations is re-expressed with respect to dimensionless space and time variables, as follows:

$$
\left\{\begin{array}{l}
\nabla_{x / L} \cdot\left(\tilde{\lambda}_{\gamma}^{*} \nabla_{x / L}\left(T_{\gamma}\right)\right)=\mathbb{D}_{\gamma_{L}} \rho_{\gamma}^{*} c_{\gamma}^{*} \frac{\partial T_{\gamma}}{\partial\left(t / t_{\mathrm{c}}\right)} \quad \text { in } \Omega_{\gamma},(\gamma=1,2), \\
T_{1}=T_{2} \quad \text { on } \Gamma, \\
\tilde{\lambda}_{1}^{*} \nabla_{x / L}\left(T_{1}\right) \cdot \mathbf{n}=\mathbb{R}_{\lambda} \tilde{\lambda}_{2}^{*} \nabla_{x / L}\left(T_{2}\right) \cdot \mathbf{n} \quad \text { on } \Gamma,
\end{array}\right.
$$

where $\mathbb{D}_{\gamma_{L}}$ and $\mathbb{R}_{\lambda}$ are dimensionless numbers, defined by:

$$
\mathbb{D}_{\gamma_{\mathrm{L}}}=\frac{L^{2} \rho_{\gamma_{\mathrm{c}}} c_{\gamma_{\mathrm{c}}}}{\lambda_{\gamma_{\mathrm{c}} t_{\mathrm{c}}}} \quad(\gamma=1,2), \quad \text { and } \quad \mathbb{R}_{\lambda}=\frac{\lambda_{2_{\mathrm{c}}}}{\lambda_{1_{\mathrm{c}}}}
$$

Since constituent 1 is connected and is further the most conductive constituent, it governs the heat transfer phenomenon. Consequently, the transient regime at the macroscopic scale $\mathrm{L}$ is reached when the characteristic time of observation, $t_{\mathrm{c}}$, is chosen of the order of $t_{\mathrm{c}}=t_{1_{\mathrm{L}}}$, where $t_{1_{\mathrm{L}}}$ denotes the macroscopic characteristic time in constituent 1:

$$
t_{1_{\mathrm{L}}}=\frac{L^{2} \rho_{1_{\mathrm{c}}} c_{1_{\mathrm{c}}}}{\lambda_{1_{\mathrm{c}}}}
$$

This leads to: $\mathbb{D}_{1_{\mathrm{L}}}=\mathscr{O}\left(\varepsilon^{0}\right)$ and $\mathbb{D}_{2_{\mathrm{L}}}=\mathscr{O}\left(\mathbb{R}_{\lambda}^{-1}\right)$. With these estimates, the dimensionless local description (2) of macroscopic transient regime only depends upon the order of magnitude of $\mathbb{R}_{\lambda}$, and indeed, the objective of the study is to identify its influence on the macroscopic behaviour. Thus, the analysis consists in sweeping all the orders of magnitude for $\mathbb{R}_{\lambda}$ and to determine the corresponding macroscopic models by homogenisation.

\subsubsection{Macroscopic models}

Four distinct macroscopic models describing transient heat transfer at the macroscopic scale (i.e. when $\mathbb{D}_{1_{\mathrm{L}}}=\mathscr{O}\left(\varepsilon^{0}\right)$ ) are obtained by homogenisation, according to the order of magnitude of $\mathbb{R}_{\lambda}$. Expressed with respect to the physical variables (i.e the usual dimensional 
variables and parameters) they read:

- Capacity model with global conductivity (I.a): $\mathbb{R}_{\lambda}=\mathscr{O}\left(\varepsilon^{0}\right)$

$$
\nabla_{x} \cdot\left(\lambda_{\left(\lambda_{1}, \lambda_{2}\right)}^{\mathrm{eff}} \nabla_{x} T\right)=\left(<\rho_{1} c_{1}>_{\Omega}^{\Omega_{1}}+<\rho_{2} c_{2}>_{\Omega}^{\Omega_{2}}\right) \frac{\partial T}{\partial t}
$$

- Capacity model with partial conductivity $(I . b): \mathbb{R}_{\lambda}=\mathscr{O}(\varepsilon)$

$$
\nabla_{x} \cdot\left(\lambda_{\left(\lambda_{1}, 0\right)}^{\mathrm{eff}} \nabla_{x} T\right)=\left(<\rho_{1} c_{1}>_{\Omega}^{\Omega_{1}}+<\rho_{2} c_{2}>_{\Omega}^{\Omega_{2}}\right) \frac{\partial T}{\partial t}
$$

- Dual-conductivity model $(I I): \mathbb{R}_{\lambda}=\mathscr{O}\left(\varepsilon^{2}\right)$

$$
\nabla_{x} \cdot\left(\lambda_{\left(\lambda_{1}, 0\right)}^{\text {eff }} \nabla_{x} T_{1}\right)=<\rho_{1} c_{1}>_{\Omega}^{\Omega_{1}} \frac{\partial T_{1}}{\partial t}+<\rho_{2} c_{2} \frac{\partial T_{2}}{\partial t}>_{\Omega}^{\Omega_{2}} \quad T_{2}=\theta * T_{1}
$$

- Single-conductivity model $(I I I): \mathbb{R}_{\lambda} \leq \mathscr{O}\left(\varepsilon^{3}\right)$

$$
\nabla_{x} \cdot\left(\lambda_{\left(\lambda_{1}, 0\right)}^{\text {eff }} \nabla_{x} T_{1}\right)=<\rho_{1} c_{1}>_{\Omega}^{\Omega_{1}} \frac{\partial T_{1}}{\partial t}
$$

Derivations of models I.a and II are detailed in (Auriault 1983).

Capacity models (I.a and I.b) are one-temperature-field models with $T=T_{1}=T_{2}$. As a consequence, the storage term is the mean value of both thermal capacities $\langle\rho c\rangle_{\Omega}$. Both capacity models are distinguished by their effective conductivity (the reader is referred to (Auriault 1983) for the definitions and the properties of effective conductivities). The global effective conductivity $\lambda_{\left(\lambda_{1}, \lambda_{2}\right)}^{\text {eff }}$ depends on both local conductivities over the whole periodic cell. For model I.b, since $\mathbb{R}_{\lambda}=\mathscr{O}\left(\lambda_{2} / \lambda_{1}\right)=\mathscr{O}(\varepsilon)$, the effective conductivity degenerates into the partial effective conductivity, $\lambda_{\left(\lambda_{1}, 0\right)}^{\text {eff }}$, that only depends upon the local conductivity of constituent 1 . Thus, both constituents contribute to the macroscopic thermal conduction in model I.a, while the less conductive constituent doesn't contribute to the macroscopic heat transfer in model I.b.

Model II is a two-temperature-field model, $T_{1} \neq T_{2}$, where only $T_{1}$ strictly varies at the macroscopic scale. Both temperature fields are linked by a convolution relationship : $T_{2}(\mathbf{x}, \mathbf{y}, t)=$ $\theta * T_{1}(\mathbf{x}, t)$. The kernel $\theta(\mathbf{y}, t)$ is the solution to the following transient thermal transfer in constituent 2 , submitted on its boundary with constituent 1 to a Dirichlet condition, namely an uniform Dirac temperature:

$$
\left\{\begin{array}{l}
\boldsymbol{\nabla}_{y} \cdot\left(\lambda_{2} \boldsymbol{\nabla}_{y} \theta\right)=\rho_{2} c_{2} \frac{\partial \theta}{\partial t} \quad \text { in } \Omega_{2}, \\
\theta(\mathbf{y}, t)=\delta(t) \quad \text { on } \Gamma, \\
\theta: \Omega \text {-periodic. }
\end{array}\right.
$$

The above model characterises a behaviour with memory effects (Auriault 1983), which is specific to the dual-conductivity behaviour. They arise macroscopically in the convoluted form involved in the effective accumulation term related to constituent $2:\left\langle\rho_{2} c_{2} \theta\right\rangle_{\Omega}^{\Omega_{2}}$ $* \frac{\partial T_{1}}{\partial t}$. Moreover, since $\mathbb{R}_{\lambda}=\mathscr{O}\left(\lambda_{2} / \lambda_{1}\right)=\mathscr{O}\left(\varepsilon^{2}\right)$, the effective conductivity is naturally $\lambda \underset{\left(\lambda_{1}, 0\right)}{\partial_{\text {eff }}}$

Model III appears as a one-temperature-field model, $T_{1}$, the temperature field of the most 
conductive constituent. In fact this is a two field situation, with $T=T_{1}$ and $T_{2}=0$. Hence $T_{2}$ is disregarded. The effective capacity $\left\langle\rho_{1} c_{1}>_{\Omega}^{\Omega_{1}}\right.$ and the effective conductivity $\lambda_{\left(\lambda_{1}, 0\right)}^{\text {eff }}$ only account for constituent 1 . Because of the extremely weak conductivity of constituent 2, $\mathbb{R}_{\lambda}=\mathscr{O}\left(\lambda_{2} / \lambda_{1}\right) \leq \mathscr{O}\left(\varepsilon^{3}\right)$, model III is the model that would be obtained if constituent 2 was a purely insulating constituent, "blind" to heat transfer occurring in constituent 1.

There exist continuous passages between these four models: model II expresses the transition between model I.b reached when $T_{1}=\mathrm{T}_{2}$, and model III is reached when $T_{2}=0$, while Model I.a converges to model I.b as $\lambda_{2}=0$.

\subsection{Interpretation of the domains of validity by means of characteristic times}

Since model II can degenerate into models I.b and III, let firstly examine its domain of validity. This model of transient transfer at the macroscopic scale, i.e. for $\mathbb{D}_{1_{\mathrm{L}}}=\mathscr{O}\left(\varepsilon^{0}\right)$, is obtained when $\mathbb{R}_{\lambda}=\mathscr{O}\left(\varepsilon^{2}\right)$. These both orders of magnitude fully characterise the domain of validity of model II. This domain of validity can thus equivalently be defined by: $\mathbb{D}_{2_{\mathrm{L}}}=$ $\mathscr{O}\left(\mathbb{D}_{1_{\mathrm{L}}}\right) \times \mathscr{O}\left(\mathbb{R}_{\lambda}^{-1}\right)=\mathscr{O}\left(\varepsilon^{-2}\right)$. From the definition of $\mathbb{D}_{2_{\mathrm{L}}}$, this gives:

$$
L^{2} \rho_{2_{\mathrm{c}}} c_{2_{\mathrm{c}}} / \lambda_{2_{\mathrm{c}}} t_{1_{\mathrm{L}}}=\mathscr{O}\left(\varepsilon^{-2}\right)=\mathscr{O}\left(L^{2} / l^{2}\right), \quad \text { hence, } \quad l^{2} \rho_{2_{\mathrm{c}}} c_{2_{\mathrm{c}}} / \lambda_{2_{\mathrm{c}}} t_{1_{\mathrm{L}}}=\mathscr{O}\left(\varepsilon^{0}\right)
$$

We note that this can be expressed as follows:

$$
\frac{t_{2_{l}}}{t_{1_{\mathrm{L}}}}=\mathscr{O}\left(\varepsilon^{0}\right), \quad \text { where } \quad t_{2_{l}}=\frac{l^{2} \rho_{2_{\mathrm{c}}} c_{2_{\mathrm{c}}}}{\lambda_{2_{\mathrm{c}}}} \quad \text { and } \quad t_{1_{L}}=\frac{L^{2} \rho_{1_{\mathrm{c}}} c_{1_{\mathrm{c}}}}{\lambda_{1_{\mathrm{c}}}} \text {, }
$$

in which $t_{2 l}$ denotes the characteristic time for transient heat conduction in constituent 2 at the local scale, characterised by $l$, while $t_{1_{\mathrm{L}}}$ is the characteristic time for transient heat conduction at the macroscopic scale, as defined by (3). Thus, the domain of validity of model II, initially defined by means of the conductivity contrast, can be equivalently characterised by (6). The fact that $t_{2_{l}}=\mathscr{O}\left(t_{1_{\mathrm{L}}}\right)$ means that two transient phenomena simultaneously occur, one at the macro-scale, and the second one at the micro-scale within the less conducting constituent.

Now, let examine the orders of magnitude of the time ratio $t_{2_{l}} / t_{1_{\mathrm{L}}}=\mathscr{O}\left(\varepsilon^{2} \mathbb{R}_{\lambda}^{-1}\right)$ obtained for the distinct transient macroscopic models. We get:

$$
\frac{t_{2_{l}}}{t_{1_{\mathrm{L}}}} \begin{cases}=\mathscr{O}\left(\varepsilon^{2}\right) & \text { for model I.a } \\ =\mathscr{O}(\varepsilon) & \text { for model I.b } \\ =\mathscr{O}\left(\varepsilon^{0}\right) & \text { for model II, } \\ \geq \mathscr{O}\left(\varepsilon^{-1}\right) & \text { for model III. }\end{cases}
$$

We thus deduce the following fundamental result that defines the domains of validity of the models by means of characteristic times:

$$
\begin{cases}\text { Capacity models (I) (I.a: } \left.\mathbb{R}_{\lambda}=\mathscr{O}\left(\varepsilon^{0}\right) ; \text { I.b: } \mathbb{R}_{\lambda}=\mathscr{O}(\varepsilon)\right): t_{2_{l}} \ll t_{1_{\mathrm{L}}} \\ \text { Dual-conductivity model (II): } \mathbb{R}_{\lambda}=\mathscr{O}\left(\varepsilon^{2}\right) & t_{2_{l}} \approx t_{1_{\mathrm{L}}} \\ \text { Single conductivity model (III): } \mathbb{R}_{\lambda} \leq \mathscr{O}\left(\varepsilon^{3}\right) & t_{2_{l}} \gg t_{1_{\mathrm{L}}}\end{cases}
$$

Noticing that $t_{2_{l}} / t_{1_{\mathrm{L}}}=\mathscr{O}\left(\varepsilon^{2} \mathbb{R}_{\lambda}^{-1}\right)$, these domains of validity can be represented on a $\log _{\varepsilon}\left(t_{2_{l}} / t_{1_{L}}\right)$ vs. $\log _{\varepsilon}\left(\mathbb{R}_{\lambda}\right)$ graph, as shown in Fig.2. It shows how the domains of validity of the homogenised models initially given by the order of magnitude of $\mathbb{R}_{\lambda}$ is translated 
into orders of magnitude of the ratio of characteristic times, $t_{2_{l}} / t_{1_{\mathrm{L}}}$. It also highlight the correspondence between the orders of the local conductivities with the effective conductivities.

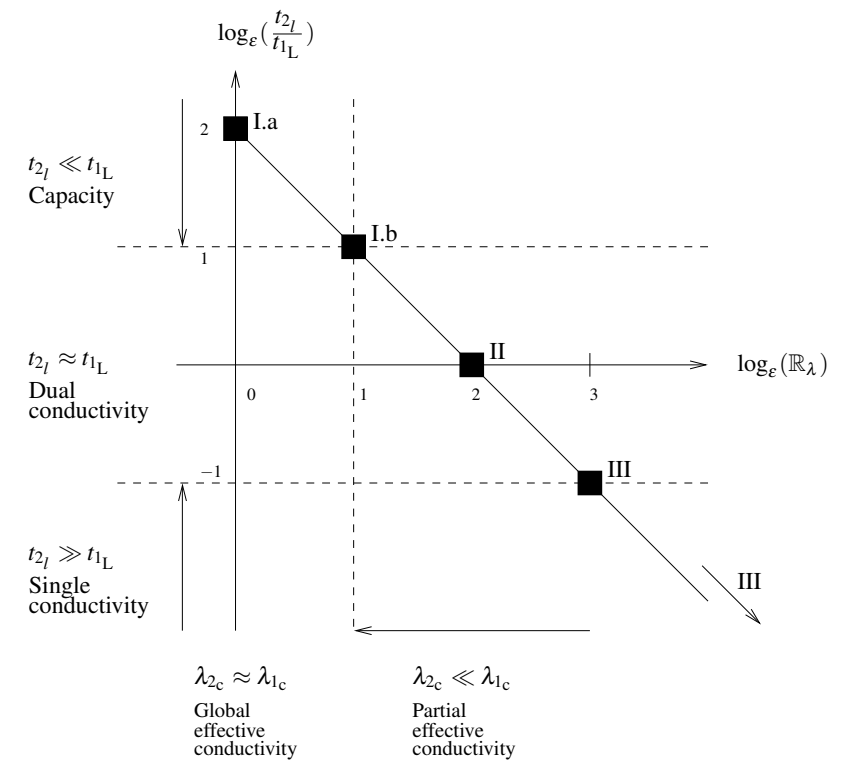

Fig. 2 Domains of validity of macroscopic models with respect to $\log _{\varepsilon}\left(t_{2_{l}} / t_{1_{\mathrm{L}}}\right)$ and $\log _{\varepsilon}\left(\mathbb{R}_{\lambda}\right)$.

We note that, in any case the following relationship is verified: $t_{1_{l}}=\varepsilon^{2} t_{1_{L}}$. This means that for the most conductive constituent (constituent 1 ), the characteristic time of observation $t_{\mathrm{c}}=\mathscr{O}\left(t_{1_{L}}\right)=\mathscr{O}\left(\varepsilon^{-2}\right) t_{1_{l}}$ is much larger than the characteristic time for transient heat conduction at the microscopic scale. Consequently, the most conductive constituent is always submitted to a quasi-steady-state regime at the local scale.

These results reveal that the macroscopic behaviour is conditioned by the local regime in the less conductive constituent 2. Since we are considering $t_{\mathrm{c}}=t_{1_{L}}$, the case $t_{2_{l}} \ll t_{1_{L}}$ means that under a macroscopic transient regime, a local quasi-steady-state regime occurs in both constituents. This leads to capacity models: there is a single temperature field i.e. a full coupling between both heat transfer processes and the influence of constituent 2 appears in the macroscopic accumulation term. When $t_{2_{l}} \approx t_{1_{L}}$, both transient processes occur simultaneously which leads to two coupled temperature fields. When $t_{2_{l}} \gg t_{1_{L}}$, we have $t_{\mathrm{c}} \ll t_{2_{l}}$, which means that for characteristic times of macroscopic transient regime, the local transient thermal conduction mechanism in constituent 2 has not started yet. In fact, it is confined into a very thin boundary layer. Constituent 2 is therefore seen as an insulating constituent.

\subsection{Order of magnitude of the flux ratio}

The purpose of this paragraph is to determine the order of magnitude of heat flux ratio $\mathbb{R}_{\mathrm{q}}=q_{2_{\mathrm{c}}} / q_{1_{\mathrm{c}}}$, where $q_{\gamma_{\mathrm{c}}}$ denotes the characteristic values of the flux defined by: $\boldsymbol{q}_{\gamma}=$ $\tilde{\lambda}_{\gamma} \boldsymbol{\nabla}\left(T_{\gamma}\right)(\gamma=1,2)$. As it will be seen in $\S 4.3$, the result is of crucial importance for the 
determination of the order of magnitude of the velocity ratio for transient fluid flow in dualporosity media. Several empirical approaches have been made for determining this order of magnitude, but with no clear understanding on the rules which condition the result (Royer and Auriault 1994), (Chastanet et al. 2007). Thanks to the above time analysis which suggests to examine the temporal regimes, we are in position to rigorously derive this fundamental result. The key points to be underlined for determining this order of magnitude are the following:

1. the order of magnitude of the ratio is distinct for a macroscopic steady-state regime and for a macroscopic transient regime;

2. under a macroscopic transient regime, the order of magnitude of the ratio is conditioned by the local temporal regimes.

Let firstly note that a given local-scale formulation of the thermal problem is defined by $\mathbb{R}_{\lambda}$ and leads to a single description at the macro-scale. Consequently, the orders of magnitude of the heat flux ratio $\mathbb{R}_{\mathrm{q}}$ and of the conductivity ratio $\mathbb{R}_{\lambda}$ are linked. The purpose is therefore to determine the relationship between $\mathbb{R}_{\mathrm{q}}$ and $\mathbb{R}_{\lambda}$. For a macroscopic steady-state regime, both temperature gradients are identical, and the order of magnitude of both fluxes are such that: $q_{1_{\mathrm{c}}}=\mathscr{O}\left(\lambda_{1_{\mathrm{c}}} T_{\mathrm{c}} / \mathrm{L}\right), q_{2_{\mathrm{c}}}=\mathscr{O}\left(\lambda_{2_{\mathrm{c}}} T_{\mathrm{c}} / \mathrm{L}\right)$, which yields $\mathbb{R}_{\mathrm{q}}=\mathscr{O}\left(\mathbb{R}_{\lambda}\right)$. Under a transient macroscopic regime, both temperature gradients may become time dependent and their orders of magnitude may no longer be identical. For determining the relationship between $\mathbb{R}_{\mathrm{q}}$ and $\mathbb{R}_{\lambda}$, the distinct local temporal regimes that have previously been identified must be distinguished. We firstly consider the case of storage models which are characterised by a local steady-state regime in both constituents $\left(t_{2} \ll t_{1_{L}}, t_{1_{l}} \ll t_{1_{L}}\right)$ and by a single temperature field $T$. Thus both temperature gradients are identical, and the order of magnitude of the flux ratio is given by $\mathbb{R}_{\mathrm{q}}=\mathscr{O}\left(\mathbb{R}_{\lambda}\right)$ as in case of a macroscopic steady-state regime. Let now consider the converse situations where $t_{2_{l}} \geq t_{1_{L}}=\mathscr{O}\left(t_{\mathrm{c}}\right)$. In these cases, the temperature gradients in both constituents are no longer of same order, because of the local transient regime in constituent 2. Consequently, the characteristic size of variation $\delta$ of temperature field $T_{2}$ is defined from the transient heat balance by:

$\frac{\rho_{2_{\mathrm{c}}} c_{2_{\mathrm{c}}} T_{2}}{t_{1_{\mathrm{L}}}}=\mathscr{O}\left(\frac{\lambda_{2_{\mathrm{c}}} T_{2}}{\delta^{2}}\right), \quad$ thus, $\quad \delta^{2}=\mathscr{O}\left(\frac{\lambda_{2_{\mathrm{c}}} t_{1_{L}}}{\rho_{2_{\mathrm{c}}} c_{2_{\mathrm{c}}}}\right)=\mathscr{O}\left(\frac{\lambda_{2_{\mathrm{c}}}}{\lambda_{1_{\mathrm{c}}}} \frac{\rho_{1_{\mathrm{c}}} c_{1_{\mathrm{c}}}}{\rho_{2_{\mathrm{c}}} c_{2_{\mathrm{c}}}}\right) \mathrm{L}^{2}$.

Hence, the characteristic value of flux $q_{2_{\mathrm{c}}}$ over interface $\Gamma$ between both constituents, and of temperature $T_{2_{\Gamma}}$ reads:

$q_{2_{\mathrm{c}}}=\mathscr{O}\left(\lambda_{2_{\mathrm{c}}} \frac{T_{2_{\Gamma}}}{\delta}\right)$

while the characteristic value of the flux in constituent 1 remains determined by:

$q_{1_{\mathrm{c}}}=\mathscr{O}\left(\lambda_{1_{\mathrm{c}}} \frac{T_{1_{\mathrm{c}}}}{L}\right)$

On the interface, the temperature continuity imposes that $T_{2_{\Gamma}}=\mathscr{O}\left(T_{1_{\mathrm{c}}}\right)$. Then, we deduce from (9) and (10), that when $t_{2_{l}} \geq t_{1_{L}}$, the order of magnitude of the flux ratio satisfies:

$$
\mathbb{R}_{\mathrm{q}}=\frac{q_{2_{\mathrm{c}}}}{q_{1_{\mathrm{c}}}}=\frac{\lambda_{2_{\mathrm{c}}}}{\lambda_{1_{\mathrm{c}}}} \frac{L}{\delta}=\sqrt{\frac{\lambda_{2_{\mathrm{c}}}}{\lambda_{1_{\mathrm{c}}}}} \sqrt{\frac{\rho_{2_{\mathrm{c}} c_{2_{\mathrm{c}}}}}{\rho_{1_{\mathrm{c}}} c_{1_{\mathrm{c}}}}}
$$

This relationship means that, in presence of local transient regime, the flux ratio is determined by the thermal impedance ratio instead of the conductivity ratio which is obtained for 
a local quasi-steady-state regime. Therefore, with the assumption $\rho_{2_{\mathrm{c}}} c_{2_{\mathrm{c}}} / \rho_{1_{\mathrm{c}}} c_{1_{\mathrm{c}}}=\mathscr{O}(1)$, the order of magnitude of the flux ratio under a macroscopic transient regime is such that:

$\mathbb{R}_{\mathrm{q}}=\frac{q_{2_{\mathrm{c}}}}{q_{1_{\mathrm{c}}}}=\left\{\begin{array}{l}\mathscr{O}\left(\mathbb{R}_{\lambda}\right)=\mathscr{O}\left(\frac{\lambda_{2_{\mathrm{c}}}}{\lambda_{1_{\mathrm{c}}}}\right) \quad \text { when } t_{2_{l}} \ll t_{1_{\mathrm{L}}}(\text { model I.a, I.b), } \\ \mathscr{O}\left(\sqrt{\mathbb{R}_{\lambda}}\right)=\mathscr{O}\left(\sqrt{\frac{\lambda_{2_{\mathrm{c}}}}{\lambda_{1_{\mathrm{c}}}}}\right) \text { when } t_{2_{l}} \geq t_{1_{\mathrm{L}}} \text { (model II, III). }\end{array}\right.$

\section{Macroscopic behaviours of dual-porosity media}

\subsection{Introduction}

The above two-scale analysis of thermal conduction in composite media allows us to interpret results obtained by using a three-scale homogenisation procedure for modelling various transfer phenomena in fluid saturated dual-porosity media. Below, we present some results that have been obtained by applying the three-scale homogenisation procedure introduced in (Auriault and Boutin 1992). We firstly briefly introduce the three-scale procedure. Then, we show that the analysis conducted for thermal conduction in composite media applies for interpretating the results obtained for compressible fluid flow in dual-porosity media. Finally, we focus on solute transport in dual-porosity media. We show that the time analysis performed for the thermal problem allows to elucidate the crucial issue of memory effects occurrence.

4.2 Three-scale homogenisation in dual-porosity media

\subsubsection{Medium description}

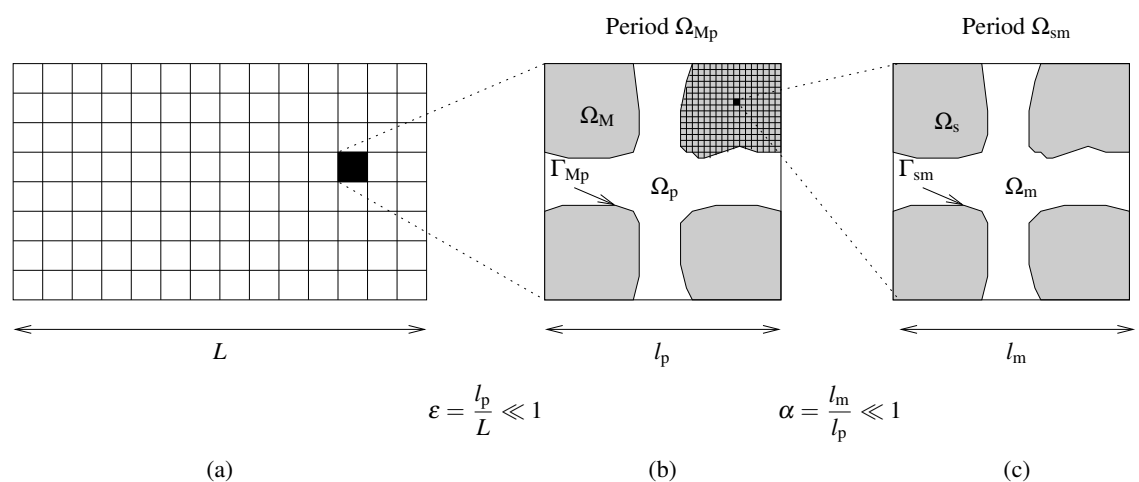

Fig. 3 Periodic dual-porosity medium: (a) Macroscopic scale; (b) Mesoscopic scale (micro-porous matrix/mesopore scale) periodic cell; (c) Microscopic scale (micro-pore scale) periodic cell.

We consider the dual-porosity medium which is sketched in Fig.3, whose characteristic macroscopic size is denoted by L. This three-scale medium consists of a micro-porous scale, 
composed of a network of meso-pores and of a micro-porous matrix, and of a microscopic scale made of a network of micro-pores and of a solid skeleton. The medium is periodic, of period $\Omega_{\mathrm{Mp}}$, and the characteristic length, $l_{\mathrm{p}}$, of this micro-porous scale is much smaller than the macroscopic characteristic length $\mathrm{L}$. We put $: \varepsilon=l_{\mathrm{p}} / \mathrm{L} \ll 1$. Periodic cell $\Omega_{\mathrm{Mp}}$ consists of the mesopore domain, $\Omega_{\mathrm{p}}$, and of the micro-porous matrix domain, $\Omega_{\mathrm{M}}$, and the interface between both domains is denoted by $\Gamma_{\mathrm{Mp}}$. The porosity related to the meso-pores is $\phi_{\mathrm{p}}=\left|\Omega_{\mathrm{p}}\right| /\left|\Omega_{\mathrm{Mp}}\right|$.

Now, the micro-porous matrix domain $\Omega_{\mathrm{M}}$ is itself periodic, of period $\Omega_{\mathrm{sm}}$ and the characteristic length of this microscopic scale, $l_{\mathrm{m}}$, is much smaller than the micro-porous length scale $l_{\mathrm{p}}$. We define: $\alpha=l_{\mathrm{m}} / l_{\mathrm{p}} \ll 1$. Periodic cell $\Omega_{\mathrm{sm}}$ consists of the micro-pore domain, $\Omega_{\mathrm{m}}$, and of the solid domain, $\Omega_{\mathrm{s}}$. Their common interface is denoted by $\Gamma_{\mathrm{sm}}$. The porosity of the micro-porous matrix is $\phi_{\mathrm{m}}=\left|\Omega_{\mathrm{m}}\right| /\left|\Omega_{\mathrm{sm}}\right|$ and the total porosity of the material is $\phi_{\mathrm{T}}=\phi_{\mathrm{p}}+\phi_{\mathrm{m}}\left(1-\phi_{\mathrm{p}}\right)$.

We introduce the following averages:

$$
<\cdot>_{\Omega_{\mathrm{sm}}}=<\cdot>_{\Omega_{\mathrm{sm}}}^{\Omega_{\mathrm{s}}}+<\cdot>_{\Omega_{\mathrm{sm}}}^{\Omega_{\mathrm{m}}}, \quad ; \quad<\cdot>_{\Omega_{\mathrm{Mp}}}=<\cdot>_{\Omega_{\mathrm{Mp}}}^{\Omega_{\mathrm{M}}}+<\cdot>_{\Omega_{\mathrm{Mp}}}^{\Omega_{\mathrm{p}}},
$$

where

$$
<\cdot>_{\Omega_{\delta}}^{\Omega_{\gamma}}=\frac{1}{\left|\Omega_{\delta}\right|} \int_{\Omega_{\gamma}} \cdot d \Omega
$$

\subsubsection{Three-scale homogenisation procedure}

The medium is therefore doubly periodic and is further characterised by two conditions of separation of scales. The condition $\varepsilon \ll 1$ is the fundamental condition of separation of scales required for applying homogenisation or any other continuum approach, while the condition $\alpha \ll 1$ ensures the dual-porosity property of the medium. For applying the threescale homogenisation procedure, the order of magnitude of $\alpha$ must be fixed. Several cases are of interest and the relevant relations between $\alpha$ and $\varepsilon$ may depend on the problem being considered. Three orders of magnitude of $\alpha$ are considered: $\alpha=\mathscr{O}\left(\varepsilon^{1 / 2}\right), \alpha=\mathscr{O}(\varepsilon)$ and $\alpha=\mathscr{O}\left(\varepsilon^{2}\right)$ (due to the condition of separation of scales $(\alpha \ll 1$ ), the case $\alpha<\mathscr{O}(\varepsilon)$ is restricted to : $\left.\alpha=\mathscr{O}\left(\varepsilon^{1 / 2}\right)\right)$.

The three-scale homogenisation procedure is performed in two steps: i) the micro-porescale description is firstly homogenised so as to determine the behaviour of the micro-porous matrix; ii) homogenisation of the micro-porous scale description which includes the mesopore equations, the homogenised description of the matrix and the mesoporepore/microporous matrix boundary-conditions is then performed and leads to the macroscopic behaviour.

\subsection{Compressible fluid flow in rigid dual-porosity media}

\subsubsection{Introduction}

Homogenisation of a highly compressible fluid through a dual-porosity medium has been investigated in (Royer and Auriault 1994). In this initial work, three models are obtained and their domains of validity are given by means of orders of magnitude of scale ratio $\alpha$ with respect to scale ratio $\varepsilon$. Furthermore, the order of magnitude of the velocity ratio was identified throughout the homogenisation process, but with no clear interpretation. 
The purpose of this section is to enhance the physical insight by clarifying the interpretation of the domains of validity and justifying the order of magnitude of the velocity ratio. These points are addressed by using the analogy with the results obtained for thermal conduction, which suggest the use of characteristic times. For the sake of simplicity, the analysis is presented for the linear problem of a compressible fluid in isothermal regime, which be extended to the case investigated in (Royer and Auriault 1994). For fluid-solid heat exchange effect one may refer to (Boutin et al. 1998).

\subsubsection{Local dimensionless governing equations}

Within both fluid domains, $\Omega_{\mathrm{m}}$ and $\Omega_{\mathrm{p}}$, fluid flow is described by Stokes equation and the mass-balance equation, where $\mu, \mathbf{v}_{\gamma}, p_{\gamma}$ and $\beta$ are the fluid viscosity, velocity, pressure and compressibility, respectively:

$$
\left\{\begin{array}{l}
\beta \frac{\partial p_{\gamma}}{\partial t}+\nabla \cdot\left(\mathbf{v}_{\gamma}\right)=0 \quad \text { in } \Omega_{\gamma}(\gamma=\mathrm{m}, \mathrm{p}) \\
\mu \Delta \mathbf{v}_{\gamma}-\nabla p_{\gamma}=\mathbf{0} \quad \text { in } \Omega_{\gamma}(\gamma=\mathrm{m}, \mathrm{p})
\end{array}\right.
$$

At the microscopic scale, we express the no-slip condition over the solid/fluid interface:

$$
\mathbf{v}_{\mathrm{m}}=\mathbf{0} \quad \text { on } \Gamma_{\mathrm{sm}} .
$$

At the micro-porous scale, the conditions over the mesopore/micro-porous matrix interface are the continuity of velocities and pressures:

$$
\left\{\begin{array}{l}
\mathbf{v}_{\mathrm{p}}=<\mathbf{v}_{\mathrm{m}}>\Omega_{\Omega_{\mathrm{sm}}}^{\Omega_{\mathrm{m}}} \\
p_{\mathrm{p}}=p_{\mathrm{m}} \quad \text { on } \Gamma_{\mathrm{Mp}}
\end{array} \text { on } \Gamma_{\mathrm{Mp}}\right.
$$

Choosing the macroscopic length $\mathrm{L}$ and a characteristic time $t_{\mathrm{c}}$ as the reference length and time respectively, this set is re-expressed with dimensionless space and time variables:

$$
\left\{\begin{array}{l}
\boldsymbol{\nabla}_{x / L} \cdot\left(\mathbf{v}_{\gamma}^{*}\right)+\mathbb{S}_{\mathrm{L}} \beta^{*} \frac{\partial p_{\gamma}^{*}}{\partial\left(t / t_{\mathrm{c}}\right)}=0 \quad \text { in } \Omega_{\gamma},(\gamma=\mathrm{m}, \mathrm{p}), \\
\mathbb{F}_{\mathrm{L}} \mu^{*} \Delta_{x / L} \mathbf{v}_{\gamma}^{*}-\nabla_{x / L}\left(p_{\gamma}^{*}\right)=\mathbf{0} \quad \text { in } \Omega_{\gamma},(\gamma=\mathrm{m}, \mathrm{p}), \\
\mathbf{v}_{\mathrm{p}}^{*}=\mathbb{R}_{\mathrm{v}}<\mathbf{v}_{\mathrm{m}}^{*}>_{\Omega_{\mathrm{sm}}} \quad ; \quad p_{\mathrm{m}}^{*}=p_{\mathrm{p}}^{*} \quad \text { on } \Gamma, \\
\mathbf{v}_{\mathrm{m}}^{*}=\mathbf{0} \quad \text { on } \Gamma_{\mathrm{sm}},
\end{array}\right.
$$

in which $\mathbb{F}_{\mathrm{L}}, \mathbb{S}_{\mathrm{L}}$, and $\mathbb{R}_{\mathrm{V}}$ denote dimensionless numbers, which are defined by:

$$
\mathbb{F}_{\mathrm{L}}=\frac{\mu_{\mathrm{c}} v_{\mathrm{c}}}{L p_{\mathrm{c}}} \quad ; \quad \mathbb{S}_{\mathrm{L}}=\frac{L \beta p_{\mathrm{c}}}{t_{\mathrm{c}} v_{\mathrm{c}}} \quad ; \quad \mathbb{R}_{\mathrm{v}}=v_{\mathrm{m}_{\mathrm{c}}} / v_{\mathrm{c}}
$$

Note that the whole consistency of the asymptotic expansions imposes an unique reference value for the variables of the same nature in both meso-pores and micro-pores. Conveniently, the reference values are the characteristic pressure and velocity in the meso-pores $\left(p_{\mathrm{c}}=p_{\mathrm{p}_{\mathrm{c}}}\right.$, $v_{\mathrm{c}}=v_{\mathrm{p}_{\mathrm{c}}}$ ). 


\subsubsection{Orders of magnitude of the dimensionless numbers}

We consider the case where a Darcy flow regime is reached at the macro-scale. This requires that the gradient of viscous forces at the pore scale be balanced by the macroscopic pressure gradient (Auriault and Sanchez-Palencia 1977), (Auriault 1991). This leads to the following equalities in the meso-pores and in the micro-pores, respectively:

$$
\begin{aligned}
\frac{\mu_{\mathrm{c}} v_{\mathrm{p}_{\mathrm{c}}}}{l_{\mathrm{p}}^{2}} & =\mathscr{O}\left(\frac{p_{\mathrm{p}_{\mathrm{c}}}}{L}\right), \\
\frac{\mu_{\mathrm{c}} v_{\mathrm{m}_{\mathrm{c}}}}{l_{\mathrm{m}}^{2}} & =\mathscr{O}(\nabla p)_{\mathrm{m}} .
\end{aligned}
$$

Equality (13) provides: $\mathbb{F}_{\mathrm{L}}=\mathscr{O}\left(l_{\mathrm{p}}^{2} / L^{2}\right)=\mathscr{O}\left(\varepsilon^{2}\right)$.

We further consider the situation of a transient macroscopic regime. This situation is reached when the time variation of the pressure is balanced by its space variation at the macro-scale. Let determine the reference time for this regime by dimensional analysis. Since the meso-pores are connected and much larger than the micro-pores, the physics is driven by the phenomenon within the meso-pores. After inserting the reference velocity identified from (13), the equality in order of magnitude of both terms from the mass balance equation in the mesopore domain provides:

$$
\mathscr{O}\left(\beta \frac{p_{\mathrm{p}_{\mathrm{c}}}}{t_{\mathrm{c}}}\right)=\mathscr{O}\left(\frac{v_{\mathrm{p}_{\mathrm{c}}}}{L}\right)=\mathscr{O}\left(\frac{p_{\mathrm{p}_{\mathrm{c}}}}{L^{2}} \frac{l_{\mathrm{p}}^{2}}{\mu_{\mathrm{c}}}\right)
$$

Thus, time and space variations of the pressure are balanced for a characteristic time of observation such that $t_{\mathrm{c}}=\mathscr{O}\left(t_{\mathrm{p}}\right)$, where :

$$
t_{\mathrm{p}_{\mathrm{L}}}=\mathscr{O}\left(\frac{L^{2} \beta}{l_{\mathrm{p}}^{2} / \mu_{\mathrm{c}}}\right)
$$

is the characteristic time for the transient regime at the macro-scale. Then, noticing that $\mathbb{F}_{\mathrm{L}} \cdot \mathbb{S}_{\mathrm{L}}=\mu \beta / t_{\mathrm{c}}=\mathscr{O}\left(l_{\mathrm{p}}^{2} / L^{2}\right)$, we deduce that $\mathbb{S}_{\mathrm{L}}=\mathscr{O}\left(\varepsilon^{0}\right)$.

We successively consider three orders of magnitude for $\alpha: \alpha=\mathscr{O}\left(\varepsilon^{1 / 2}\right), \alpha=\mathscr{O}(\varepsilon)$ and $\alpha=\mathscr{O}\left(\varepsilon^{2}\right)$. In steady-state regime, since $\mu_{\mathrm{c}} v_{\mathrm{m}_{\mathrm{c}}} / l_{\mathrm{m}}^{2}=\mathscr{O}\left(p_{\mathrm{c}} / L\right)$ and $\mu_{\mathrm{c}} v_{\mathrm{p}_{\mathrm{c}}} / l_{\mathrm{p}}^{2}=\mathscr{O}\left(p_{\mathrm{c}} / L\right)$, the order of magnitude of the velocity ratio is: $\mathbb{R}_{\mathrm{v}}=v_{\mathrm{m}_{\mathrm{c}}} / v_{\mathrm{p}_{\mathrm{c}}}=l_{\mathrm{m}}^{2} / l_{\mathrm{p}}^{2}=\mathscr{O}\left(\alpha^{2}\right)$. This estimate is no longer valid in transient regime. The order of magnitude of $\mathbb{R}_{\mathrm{V}}$ can be obtained on a trial-and-error basis, thanks to the fact that if a wrong order of magnitude of $\mathbb{R}_{V}$ is considered, the homogenisation calculations can't be achieved. We find that it depends on the order of magnitude of $\alpha:^{1}$

$$
\left\{\begin{array}{l}
\mathbb{R}_{\mathrm{V}}=\mathscr{O}(\varepsilon) \text { when } \alpha=\mathscr{O}\left(\varepsilon^{1 / 2}\right), \\
\mathbb{R}_{\mathrm{V}}=\mathscr{O}(\varepsilon) \text { when } \alpha=\mathscr{O}(\varepsilon), \\
\mathbb{R}_{\mathrm{V}}=\mathscr{O}\left(\varepsilon^{2}\right) \text { when } \alpha=\mathscr{O}\left(\varepsilon^{2}\right) .
\end{array}\right.
$$

\footnotetext{
${ }^{1}$ In (Royer and Auriault 1994), the flux ratio was defined on the basis of mean values of the velocities in the domains instead of values on the boundary $\Gamma$. For this reason, when $\alpha=\mathscr{O}\left(\varepsilon^{2}\right)$, the velocity ratio was found to be $\mathscr{O}\left(\varepsilon^{3}\right)$.
} 


\subsubsection{Macroscopic models}

Applying the homogenisation procedure for each of the three orders of $\alpha$ leads to the three following first-order macroscopic models:

- Model with reservoir effect $(I): \alpha=\mathscr{O}\left(\varepsilon^{1 / 2}\right)$

$$
\nabla_{x} \cdot\left(\frac{\tilde{K}_{\mathrm{p}}}{\mu} \nabla p\right)=\left(\phi_{\mathrm{p}}+\phi_{\mathrm{m}}\left(1-\phi_{\mathrm{p}}\right)\right) \beta \frac{\partial p}{\partial t}
$$

- Dual-porosity model (II): $\alpha=\mathscr{O}(\varepsilon)$

$$
\nabla_{x} \cdot\left(\frac{\tilde{K}_{\mathrm{p}}}{\mu} \nabla p_{\mathrm{p}}\right)=\phi_{\mathrm{p}} \beta \frac{\partial p_{\mathrm{p}}}{\partial t}+\phi_{\mathrm{m}} \beta \frac{\partial<p_{\mathrm{m}}>_{\Omega_{\mathrm{Mp}}}^{\Omega_{\mathrm{M}}}}{\partial t} \quad p_{\mathrm{m}}=\pi * p_{\mathrm{m}}
$$

- Single-porosity model (III): $\alpha=\mathscr{O}\left(\varepsilon^{2}\right)$

$$
\boldsymbol{\nabla}_{x} \cdot\left(\frac{\tilde{K}_{\mathrm{p}}}{\mu} \nabla p_{\mathrm{p}}\right)=\phi_{\mathrm{p}} \beta \frac{\partial p_{\mathrm{p}}}{\partial t}
$$

where $\tilde{K}_{\mathrm{p}}$ represents the permeability of the mesopore network.

Model (I) is a one-pressure-field model, i.e. $p=p_{\mathrm{m}}=p_{\mathrm{p}}$. This is an apparent singleporosity model, in which the effective permeability is the mesopore permeability, while the effective porosity is that of the whole dual-porosity medium. We designate this property by "reservoir effect" to signify that the micro-porous matrix plays the role of a fluid reservoir.

Model (II) is a two-pressure-field model $\left(p_{\mathrm{m}} \neq p_{\mathrm{p}}\right)$, which is a characteristic of a dualporosity behaviour. The notable property is that the pressure field in the micro-porous matrix is not uniform. It is related to the pressure in the meso-pores by the convolution relationship: $p_{\mathrm{m}}(\mathbf{x}, \mathbf{y}, t)=\pi * p_{\mathrm{p}}(\mathbf{x}, t)$, which introduces memory effects. The kernel $\pi(\mathbf{y}, t)$ is the solution to the transient pressure diffusion problem in the micro-porous domain submitted on its boundary with the micro-pore domain to a Dirichlet condition, in the form of a uniform Dirac pressure:

$$
\left\{\begin{array}{l}
\nabla_{y} \cdot\left(\frac{\tilde{K}_{\mathrm{m}}}{\mu} \nabla_{y} \pi\right)=\phi_{\mathrm{m}} \beta \frac{\partial \pi}{\partial t} \quad \text { in } \Omega_{\mathrm{M}} \\
\pi=\delta(t) \quad \text { on } \Gamma_{\mathrm{Mp}}, \\
\pi: \Omega_{\mathrm{Mp}} \text {-periodic, }
\end{array}\right.
$$

where $\tilde{K}_{\mathrm{m}}$ is the permeability of the micro-porous matrix.

Model III describes the flow in the mesopore network with an apparent impervious and non-porous matrix. It is therefore a single-porosity model, precisely the model that would results from the mesopore network only.

\subsection{Analogy with thermal conduction in dual-conductivity media}

Despite the significant differences in the physics of mass transfer (governed by Stokes law) and of heat transfer (governed by Fourier's law), we observe a close similarity between the different macroscopic models derived for both phenomena (see models I.b, II and III 
obtained in $\$ 3.2 .3$ ). The reason lies in the fact that upscaling Stokes law leads to Darcy's law, which is of identical diffusion type to Fourier's law. This remark enables to establish a strict analogy between temperature, heat flux, conductivity, thermal capacity, and pressure, velocity, permeability, compressibility, respectively. At the macro-scale, everything happens just as if the micro-porous matrix was a medium of permeability $K_{\mathrm{m}}$ and if the mesopore network was a medium of permeability $K_{\mathrm{p}}$. A direct consequence of this analogy is that the above models also apply for dual-permeability media, each domain being specified by its permeability without explicit description of the corresponding pore networks. This case requires a simpler treatment since only two scales are involved.

Note however that, in accordance with (13) and (14), the permeabilities are related to the meso-pores and micro-pores sizes by $K_{\gamma}=l_{\gamma}^{2} / \mu(\gamma=\mathrm{m}, \mathrm{p})$. Hence, the condition of separation between the mesopore and the micro-pore scales requires a permeability contrast. For this reason, model I.a determined for heat transfer is not obtained for mass transfer in dual-porosity media. Let finally underline that the permeability ratio is such that: $K_{\mathrm{m}} / K_{\mathrm{p}}=$ $\left(l_{\mathrm{m}} / l_{\mathrm{p}}\right)^{2}=\alpha^{2}$. Consequently, the classification of mass transfer model I, II and III is the exact transposition of the classification of heat transfer models I.b, II and III.

\subsection{Interpretation in terms of characteristic times}

\subsubsection{Domains of validity}

The domains of validity of the models are such that:

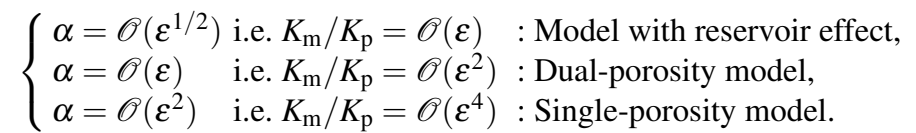

By similarity with the ratio $t_{2_{l}} / t_{1_{L}}$ in thermal conduction, we examine the order of magnitude of the ratio $t_{\mathrm{M}_{l \mathrm{p}}} / t_{\mathrm{p}_{\mathrm{L}}}$, where $t_{\mathrm{M}_{l \mathrm{p}}}$ and $t_{\mathrm{p}_{\mathrm{L}}}$ are the characteristic times for local transient diffusion regime in the micro-porous matrix and at the macroscopic scale, respectively. This latter time has been already assessed in the previous section. Meanwhile, $t_{\mathrm{M}_{l_{\mathrm{p}}}}$ and $t_{\mathrm{p}_{\mathrm{L}}}$ can also be estimated considering separately the micro-porous matrix on the one hand, and the network of meso-pores in an impermeable matrix on the second hand. Let consider the mass-balance equation which describes compressible fluid flow in a single porosity medium:

$\phi \beta \frac{\partial p}{\partial t}-\nabla \cdot\left(\frac{\tilde{K}}{\mu} \nabla p\right)=0$.

By dimensional analysis, we deduce the characteristic time for a transient diffusion regime in a domain of size $l_{\mathrm{c}}: t_{l_{\mathrm{c}}}=\phi \beta \mu l_{\mathrm{c}}^{2} / K_{\mathrm{c}}$. Thus, considering the meso-size $l_{\mathrm{p}}$ for the microporous domain and the macro-size $L$ for the porous domain, we deduce that:

$$
t_{\mathrm{M}_{l \mathrm{p}}}=\phi_{\mathrm{m}} \beta \mu l_{\mathrm{p}}^{2} / K_{\mathrm{m}_{\mathrm{c}}} \quad ; \quad t_{\mathrm{p}_{\mathrm{L}}}=\phi_{\mathrm{p}} \beta \mu L^{2} / K_{\mathrm{p}_{\mathrm{c}}} \quad ; \quad \text { hence } \quad \frac{t_{\mathrm{M}_{l \mathrm{p}}}}{t_{\mathrm{p}_{\mathrm{L}}}}=\mathscr{O}\left(\frac{\phi_{\mathrm{m}}}{\phi_{\mathrm{p}}} \frac{K_{\mathrm{p}_{\mathrm{c}}}}{K_{\mathrm{m}_{\mathrm{c}}}} \frac{l_{\mathrm{p}}^{2}}{L^{2}}\right) .
$$

Assuming that both porosities are of same order with respect to $\varepsilon, \phi_{\mathrm{M}} / \phi_{\mathrm{p}}=\mathscr{O}\left(\varepsilon^{0}\right)$, and since the order of the permeability ratio satisfies $K_{\mathrm{M}_{\mathrm{c}}} / K_{\mathrm{p}_{\mathrm{c}}}=\mathscr{O}\left(l_{\mathrm{m}}^{2} / l_{\mathrm{p}}^{2}\right)=\mathscr{O}\left(\alpha^{2}\right)$, we deduce:

$$
\frac{t_{\mathrm{M} / \mathrm{p}}}{t_{\mathrm{p}_{\mathrm{L}}}}=\mathscr{O}\left(\alpha^{-2} \varepsilon^{2}\right)\left\{\begin{array}{l}
<\mathscr{O}\left(\varepsilon^{0}\right) \text { when } \alpha>\mathscr{O}(\varepsilon), \\
=\mathscr{O}\left(\varepsilon^{0}\right) \text { when } \alpha=\mathscr{O}(\varepsilon), \\
>\mathscr{O}\left(\varepsilon^{0}\right) \text { when } \alpha<\mathscr{O}(\varepsilon),
\end{array}\right.
$$


which confirms that the three orders of $\alpha\left(\alpha=\mathscr{O}\left(\varepsilon^{1 / 2}\right), \alpha=\mathscr{O}(\varepsilon)\right.$ and $\left.\alpha=\mathscr{O}\left(\varepsilon^{2}\right)\right)$ are relevant to distinguish the three types of behaviour. Note also that this result indicates that the single porosity model III is already reached when $\alpha=\left(\varepsilon^{3 / 2}\right)$. Hence, the definition of domains of validity (18) can be improved by:

$\left\{\begin{array}{l}t_{\mathrm{M}_{l_{\mathrm{p}}}} \ll t_{\mathrm{pL}} \text { Model with reservoir effect, } \\ t_{\mathrm{M}_{\mathrm{lp}}} \approx t_{\mathrm{pL}} \text { Dual-porosity model, } \\ t_{\mathrm{M}_{\mathrm{p}}} \gg t_{\mathrm{pL}} \text { Single-porosity model. }\end{array}\right.$

\subsubsection{Velocity ratio}

Finally, the order of magnitude of the velocity ratio can be deduced by the same reasoning than that developed for the thermal problem. Let recall that the macroscopic transient regime is characterised by $t_{\mathrm{p}_{L}}$ and that the velocities are of the order of $v_{\gamma_{\mathrm{c}}}=\mathscr{O}\left(K_{\gamma_{\mathrm{c}}} / \phi_{\gamma} \boldsymbol{\nabla}\left(p_{\gamma}\right)\right)$ $(\gamma=\mathrm{m}, \mathrm{p})$. When both constituents undergo a local steady-state regime $\left(t_{\mathrm{M}_{l_{p}}} \ll t_{\mathrm{p}_{L}}, t_{\mathrm{p}_{p}} \ll\right.$ $t_{\mathrm{p}_{L}}$ ), a single pressure field $p$ arises and both pressure gradients are identical. Hence, the flux ratio $\mathbb{R}_{\mathrm{v}}$ is equal to the permeability ratio divided by the porosity ratio, i.e.: $v_{\mathrm{m}_{\mathrm{c}}} / v_{\mathrm{p}_{\mathrm{c}}}=$ $\mathscr{O}\left(\tilde{K}_{\mathrm{m}_{\mathrm{c}}} \phi_{p} / \tilde{K}_{\mathrm{p}_{\mathrm{c}}} \phi_{m}\right)=\mathscr{O}\left(l_{\mathrm{m}}^{2} \phi_{p} / l_{\mathrm{p}}^{2} \phi_{m}\right)$, which is in agreement with (13) and (14). Now, if the micro-porous domain undergoes a transient regime $\left(t_{\mathrm{M}_{l_{p}}} \geq t_{\mathrm{p}_{L}}\right)$, the pressure gradients in the meso-pores and in the micro-pores are of different orders. The characteristic size of variation $\delta$ of $p_{m}$ is defined from the transient mass balance (19):

$\frac{\phi_{m} \beta}{t_{\mathrm{p}_{L}}}=O\left(\frac{\tilde{K}_{m}}{\mu \delta^{2}}\right), \quad$ thus, $\quad \delta^{2}=\mathscr{O}\left(\frac{\tilde{K}_{m_{\mathrm{c}}}}{\mu} \frac{t_{\mathrm{p}_{\mathrm{L}}}}{\phi_{m} \beta}\right)=\mathscr{O}\left(\frac{\tilde{K}_{m_{\mathrm{c}}}}{\tilde{K}_{p_{\mathrm{c}}}} \frac{\phi_{p}}{\phi_{m}}\right) L^{2}$

Consequently, the order of magnitude of the flux ratio is in agreement with (13) and (14) and reads:

$\mathbb{R}_{\mathrm{v}}=\frac{v_{\mathrm{m}_{\mathrm{c}}}}{v_{\mathrm{p}_{\mathrm{c}}}}=\mathscr{O}\left(\frac{\tilde{K}_{m}}{\tilde{K}_{p}} \frac{L}{\delta}\right)=\sqrt{\frac{\tilde{K}_{m}}{\tilde{K}_{p}}} \sqrt{\frac{\phi_{m}}{\phi_{p}}}$

This leads to the direct translation of (11) for the pressure diffusion:

$\mathbb{R}_{\mathrm{v}}=\left\{\begin{array}{l}=\mathscr{O}\left(\frac{K_{\mathrm{m}_{\mathrm{c}}}}{K_{\mathrm{p}_{\mathrm{c}}}} \frac{\phi_{m}}{\phi_{p}}\right) \quad \text { when } t_{\mathrm{m}_{\mathrm{p}}} \ll t_{\mathrm{p}_{\mathrm{L}}}, \\ =\mathscr{O}\left(\sqrt{\frac{K_{\mathrm{m}_{\mathrm{c}}}}{K_{\mathrm{p}_{\mathrm{c}}}}} \sqrt{\frac{\phi_{m}}{\phi_{p}}}\right) \text { if } t_{\mathrm{m}_{\mathrm{p}}} \geq t_{\mathrm{p}_{\mathrm{L}}},\end{array}\right.$

or equivalently,

$\mathbb{R}_{\mathrm{v}}=\left\{\begin{array}{l}=\mathscr{O}\left(\alpha^{2}\right) \text { if } \alpha>\mathscr{O}(\varepsilon) \\ =\mathscr{O}(\alpha) \text { if } \alpha \leq \mathscr{O}(\varepsilon)\end{array}\right.$

This provides a justification of the orders of magnitude (15). 
4.6 Solute transport by diffusion - dispersion in dual-porosity media

\subsubsection{Introduction}

Fickean diffusion in composite media presents a strict analogy with thermal diffusion (Auriault and Lewandowska 1995): temperature, heat flux, conductivity and thermal capacity are replaced by solute concentration, solute flux, diffusivity and porosity, respectively. In dual-porosity media, when solute transfer results only from a purely diffusive process, the dual-conductivity model established for thermal transfer also applies, but the diffusion of constituent 2 is the effective diffusion in the micro-porous matrix. Therefore, the high contrast of diffusion required for dual-diffusion (model II) can only occur in the particular case of a highly tortuous micro-porosity. This may happen but doesn't fit with the situations that are reported in the literature, related to memory effects for solute transport in dual-porosity media. This remark suggests that an other mechanism is involved. These memory effects during solute transport are usually attributed to diffusion mechanism in the micro-pores while dispersion occurs in the meso-pores. We therefore focus the analysis on this combination of regimes. The main objective of this section is at least to partially clarify the key issue of memory effects occurrence during solute transport by diffusion in the micro-pores and dispersion in the meso-pores.

\subsubsection{Local governing equations}

Let consider the dual-porosity medium depicted in Fig.3. At the microscopic scale, we consider a purely diffusive solute transport regime. The equations read:

$$
\left\{\begin{array}{l}
\frac{\partial c_{\mathrm{m}}}{\partial t}-\boldsymbol{\nabla} \cdot\left(D \boldsymbol{\nabla} c_{\mathrm{m}}\right)=0 \quad \text { in } \Omega_{\mathrm{m}} \\
\left(D \boldsymbol{\nabla} c_{\mathrm{m}}\right) \cdot \mathbf{n}_{\mathrm{sm}}=0 \quad \text { on } \Gamma_{\mathrm{sm}}
\end{array}\right.
$$

where $c_{\mathrm{m}}$ denotes the solute concentration and $D$ represents the coefficient of molecular diffusion. At the micro-porous scale, we consider the convection-diffusion equation in the fluid phase and the boundary-conditions over the mesopore/micro-porous matrix interface are the no-slip condition and the continuity of velocities and concentrations:

$$
\left\{\begin{array}{l}
\frac{\partial c_{\mathrm{p}}}{\partial t}-\boldsymbol{\nabla} \cdot\left(D \boldsymbol{\nabla} c_{\mathrm{p}}-c_{\mathrm{p}} \mathbf{v}_{\mathrm{p}}\right)=0 \quad \text { in } \Omega_{\mathrm{p}} \\
\mathbf{v}_{\mathrm{p}}=0 \quad \text { on } \Gamma_{\mathrm{Mp}}, \\
\left(D \boldsymbol{\nabla} c_{\mathrm{p}}\right) \cdot \mathbf{n}_{\mathrm{Mp}}=<D \nabla c_{\mathrm{m}}>_{\Omega_{\mathrm{sm}}} \cdot \mathbf{n}_{\mathrm{Mp}} \quad \text { on } \Gamma_{\mathrm{Mp}}, \\
c_{\mathrm{p}}=c_{\mathrm{m}} \quad \text { on } \Gamma_{\mathrm{Mp}},
\end{array}\right.
$$

where $c_{\mathrm{p}}$ and $\mathbf{v}_{\mathrm{p}}$ denote the solute concentration and the fluid velocity.

Choosing the macroscopic length $\mathrm{L}$ and a characteristic time $t_{\mathrm{c}}$ as the characteristic length and time of reference, respectively, the above equations can be cast in dimensionless form 
as follows:

$$
\left\{\begin{array}{l}
\mathbb{D}_{\mathrm{L}} \frac{\partial c_{\mathrm{m}}^{*}}{\partial t / t_{\mathrm{c}}}-\nabla_{x / L} \cdot\left(D^{*} \nabla_{x / L} c_{\mathrm{m}}^{*}\right)=0 \quad \text { in } \Omega_{\mathrm{m}}, \\
\left(D^{*} \nabla_{x / L} c_{\mathrm{m}}^{*}\right) \cdot \mathbf{n}_{\mathrm{sm}}=0 \quad \text { on } \Gamma_{\mathrm{sm}}, \\
\mathbb{D}_{\mathrm{L}} \frac{\partial c_{\mathrm{p}}^{*}}{\partial t / t_{\mathrm{c}}}-\nabla_{x / L} \cdot\left(D^{*} \nabla_{x / L} c_{\mathrm{p}}^{*}-\mathbb{P e}_{\mathrm{p} \mathrm{L}} c_{\mathrm{p}}^{*} \mathbf{v}_{\mathrm{p}}^{*}\right)=0 \quad \text { in } \Omega_{\mathrm{p}} \\
\mathbf{v}_{\mathrm{p}}^{*}=0 \quad \text { on } \Gamma_{\mathrm{Mp}}, \\
\left(D^{*} \nabla_{x / l} c_{\mathrm{p}}^{*}\right) \cdot \mathbf{n}_{\mathrm{Mp}}=\mathbb{R}_{\mathrm{D}}<D^{*} \nabla_{x / L} c_{\mathrm{m}}^{*}>_{\Omega_{\mathrm{m}}} \cdot \mathbf{n}_{\mathrm{Mp}} \quad \text { on } \Gamma_{\mathrm{Mp}}, \\
c_{\mathrm{p}}^{*}=c_{\mathrm{m}}^{*} \quad \text { on } \Gamma_{\mathrm{Mp}} .
\end{array}\right.
$$

The dimensionless parameters $\mathbb{D}_{\mathrm{L}}, \mathbb{R}_{\mathrm{D}}$ and the Péclet number $\mathbb{P e}_{\mathrm{p}_{\mathrm{L}}}$ are defined by: $\mathbb{D}_{\mathrm{L}}=$ $\mathrm{L}^{2} / D_{\mathrm{c}} t_{\mathrm{c}}, \mathbb{R}_{\mathrm{D}}=D_{\mathrm{M}_{\mathrm{c}}}^{\mathrm{D}} / D_{\mathrm{c}}$ and $\mathbb{P e}_{\mathrm{p}_{\mathrm{L}}}=v_{\mathrm{p}_{\mathrm{c}}} \mathrm{L} / D_{\mathrm{c}}$.

\subsubsection{Orders-of-magnitude of dimensionless numbers}

The macroscopic transport regime is conditioned by the transport regime which is reached within the network of meso-pores, and accordingly to (Auriault and Adler 1995), it is:

$$
\left\{\begin{array}{lr}
\text { purely diffusive } & \text { when } \mathbb{P e}_{\mathrm{p}_{\mathrm{L}}} \leq \mathscr{O}(\varepsilon), \\
\text { advective-diffusive } & \text { when } \mathrm{Pe}_{\mathrm{p}_{\mathrm{L}}}=\mathscr{O}\left(\varepsilon^{0}\right), \\
\text { dispersive } & \text { when } \mathrm{Pe}_{\mathrm{p}_{\mathrm{L}}}=\mathscr{O}\left(\varepsilon^{-1}\right), \\
\text { strongly dispersive with no homogenised model when } \mathrm{Pe}_{\mathrm{p}_{\mathrm{L}}} \geq \mathscr{O}\left(\varepsilon^{-2}\right)
\end{array}\right.
$$

We therefore focus the analysis on the cases where $\mathbb{P} e_{\mathrm{p}_{\mathrm{L}}} \geq \mathscr{O}\left(\varepsilon^{-1}\right)$. Péclet's number in the meso-pores can be expressed as the ratio of the two following characteristic times: $\mathbb{P e}_{\mathrm{p}_{\mathrm{L}}}=$ $t_{\mathrm{L}}^{\mathrm{D}} / t_{\mathrm{L}}^{\mathrm{C}}$, where $t_{\mathrm{L}}^{\mathrm{D}}=L^{2} / D_{c}$ is the macroscopic characteristic time of diffusion, and $t_{\mathrm{L}}^{\mathrm{C}}=L / v_{\mathrm{p}_{\mathrm{c}}}$ is the macroscopic characteristic time of convection. In dispersive regime $\left(\mathbb{P e}_{\mathrm{p}_{\mathrm{L}}} \geq \mathscr{O}\left(\varepsilon^{-1}\right)\right)$, we have $t_{\mathrm{L}}^{\mathrm{C}}<t_{\mathrm{L}}^{\mathrm{D}}$, which highlights the fact that the convective transport is the preponderant transport mechanism. To obtain a macroscopic transient regime, we thus take $t_{\mathrm{c}}=\mathscr{O}\left(t_{\mathrm{L}}^{\mathrm{C}}\right)$ which leads to $\mathbb{D}_{\mathrm{L}}=\mathscr{O}\left(\varepsilon^{-1}\right)$. The characteristic value of the tensor of effective diffusion in the micro-porous matrix is such that: $D_{\mathrm{M}_{\mathrm{c}}}^{\mathrm{D}}=\mathscr{O}\left(\phi_{\mathrm{m}} T_{\mathrm{m}_{\mathrm{c}}} D_{c}\right)$, where $T_{\mathrm{m}_{\mathrm{c}}}$ is the characteristic value of the tensor of tortuosity. We thus have $\mathbb{R}_{\mathrm{D}}=D_{\mathrm{M}_{\mathrm{c}}}^{\mathrm{D}} / D_{\mathrm{c}}=\mathscr{O}\left(\phi_{\mathrm{m}} T_{\mathrm{m}_{\mathrm{c}}}\right)$. Note that in the most general cases, we have: $\phi_{\mathrm{m}} T_{\mathrm{m}_{\mathrm{c}}}=\mathscr{O}\left(\varepsilon^{0}\right)$, which leads to: $\mathbb{R}_{\mathrm{D}}=\mathscr{O}\left(\varepsilon^{0}\right)$. Lower orders of magnitude of $\mathbb{R}_{\mathrm{D}}$ correspond to highly tortuous porous media. Note that the results reported in the literature mention the occurrence of memory effects in media for which $\mathbb{R}_{\mathrm{D}}=\mathscr{O}\left(\varepsilon^{0}\right)$.

\subsubsection{Analysis of the characteristic times and identification of three case studies}

Since the transport is purely diffusive in the micro-pores and dispersive in the meso-pores, characteristic times $t_{\mathrm{M}_{\mathrm{lp}}}$ and $t_{\mathrm{p}_{\mathrm{L}}}$ are defined by

$$
t_{\mathrm{M}_{l \mathrm{p}}}=\frac{\phi_{\mathrm{M}_{\mathrm{c}}} l_{\mathrm{p}_{\mathrm{c}}}^{2}}{D_{\mathrm{M}_{\mathrm{c}}}^{\mathrm{D}}}, \quad t_{\mathrm{p}_{\mathrm{L}}}=\frac{\phi_{\mathrm{p}_{\mathrm{c}}} L}{V_{\mathrm{p}_{\mathrm{c}}}} .
$$

The ratio is expressed as:

$$
\frac{t_{\mathrm{M}_{\mathrm{p}}}}{t_{\mathrm{p}_{\mathrm{L}}}}=\mathscr{O}\left(\frac{\phi_{\mathrm{M}_{\mathrm{c}}}}{\phi_{\mathrm{p}_{\mathrm{c}}}}\right) \times \mathscr{O}\left(\frac{D_{\mathrm{p}_{c}}^{\mathrm{H}}}{D_{\mathrm{M}_{\mathrm{c}}}^{\mathrm{D}}}\right) \times \mathscr{O}\left(\varepsilon^{2}\right) \times \mathscr{O}\left(P \mathrm{e}_{\mathrm{p}_{\mathrm{L}}}\right)=\mathscr{O}\left(\mathbb{R}_{\mathrm{D}}\right) \times \mathscr{O}\left(\varepsilon^{2}\right) \times \mathscr{O}\left(P \mathrm{e}_{\mathrm{p}_{\mathrm{L}}}\right) .
$$


Let firstly consider $\mathbb{R}_{\mathrm{D}}=\mathscr{O}\left(\varepsilon^{0}\right)$. The characteristic time ratio is such that:

$$
\frac{t_{\mathrm{M}_{l_{\mathrm{p}}}}}{t_{\mathrm{p}_{\mathrm{L}}}}=\left\{\begin{array}{l}
\mathscr{O}(\varepsilon) \text { when } \mathbb{P e}_{\mathrm{p}_{\mathrm{L}}}=\mathscr{O}\left(\varepsilon^{-1}\right), \\
\mathscr{O}\left(\varepsilon^{0}\right) \text { when } \mathbb{P e}_{\mathrm{p}_{\mathrm{L}}}=\mathscr{O}\left(\varepsilon^{-2}\right)
\end{array}\right.
$$

Now, let assume that the order of magnitude $\mathbb{R}_{\mathrm{D}}=\mathscr{O}(\varepsilon)$ can be reached. This may happen in the case of a very tortuous medium for which: $\phi_{\mathrm{m}} T_{\mathrm{m}_{\mathrm{c}}}=\mathscr{O}(\varepsilon)$. In that case, we note that:

$$
\frac{t_{\mathrm{M}_{l_{\mathrm{p}}}}}{t_{\mathrm{p}_{\mathrm{L}}}}=\mathscr{O}\left(\varepsilon^{0}\right) \text { when } \mathbb{P e}_{\mathrm{p}_{\mathrm{L}}}=\mathscr{O}\left(\varepsilon^{-1}\right) .
$$

Let focus the analysis on these three above defined cases:

$$
\begin{cases}\bullet i) & \mathbb{R}_{\mathrm{D}}=\mathscr{O}\left(\varepsilon^{0}\right) \mathbb{P e}_{\mathrm{p}_{\mathrm{L}}}=\mathscr{O}\left(\varepsilon^{-1}\right) \\ \bullet i i) & \mathbb{R}_{\mathrm{D}}=\mathscr{O}\left(\varepsilon^{0}\right) \mathbb{P e}_{\mathrm{p}_{\mathrm{L}}}=\mathscr{O}\left(\varepsilon^{-2}\right) \\ \bullet i i i) & \mathbb{R}_{\mathrm{D}}=\mathscr{O}(\varepsilon) \mathbb{P e}_{\mathrm{p}_{\mathrm{L}}}=\mathscr{O}\left(\varepsilon^{-1}\right)\end{cases}
$$

From the results established in terms of characteristic times for thermal conduction, we can predict that the macroscopic behaviour is with reservoir effect in case i) and with memory effects in cases ii) and iii).

\subsubsection{Case studies}

i) $\mathbb{R}_{\mathrm{D}}=\mathscr{O}\left(\varepsilon^{0}\right)$ and $\mathbb{P e}_{\mathrm{p}_{\mathrm{L}}}=\mathscr{O}\left(\varepsilon^{-1}\right)$

Homogenisation of this case has been performed in (Royer et al. 2002), (Tejchman 2004) and leads to the following second-order model:

$\phi_{\mathrm{T}} \frac{\partial c}{\partial t}-\nabla_{x} \cdot\left(\tilde{D}^{\mathrm{H}} \nabla_{x} c-c \mathbf{V}_{\mathrm{p}}\right)=0$

This transport model is a single-continuum model: the whole dual-porosity medium is seen as an equivalent single-porosity medium. Tensor $\tilde{D}^{\mathrm{H}}$ denotes the tensor of hydrodynamic dispersion and is defined over the dual-porosity medium, while $\mathbf{V}_{\mathrm{p}}$ represents the macroscopic fluid velocity in the macro-pore network. It is a model with reservoir effect which corresponds to model I.a obtained in thermal conduction. Therefore, it doesn't reproduce the experimentally observed coupling effects.

ii) $\mathbb{R}_{\mathrm{D}}=\mathscr{O}\left(\varepsilon^{0}\right)$ and $\mathbb{P e}_{\mathrm{p}_{\mathrm{L}}}=\mathscr{O}\left(\varepsilon^{-2}\right)$

At this high magnitude of the Péclet number, the local transport mechanism in the macropores is convection-dominated and it has been shown in (Auriault and Adler 1995) that there exists no homogenised description in that case. This means that when $\mathbb{R}=\mathscr{O}\left(\varepsilon^{0}\right)$ and $P \mathrm{e}_{\mathrm{p}_{\mathrm{L}}}=\mathscr{O}\left(\varepsilon^{-2}\right)$, memory effects occur but which cannot be modelled in the framework of an equivalent continuous macroscopic description. It seems that most of the memory effects reported in the literature and which are modelled by means of the so-called mobile-immobile (MIM) two-equation model of Barenblatt's type might correspond to these orders of magnitude. Note that in this case, the equality of the characteristic times allows to conclude that the apparent behaviour presents memory effects, while there exists no continuum model for reproducing this behaviour. 
iii) $\mathbb{R}_{\mathrm{D}}=\mathscr{O}(\varepsilon)$ and $\mathbb{P e}_{\mathrm{p}_{\mathrm{L}}}=\mathscr{O}\left(\varepsilon^{-1}\right)$.

It can be shown that the corresponding first-order macroscopic model reads:

$\phi_{\mathrm{p}} \frac{\partial c_{\mathrm{p}}}{\partial t}+\frac{\partial<c_{\mathrm{m}}>_{\Omega_{\mathrm{Mp}}}^{\Omega_{\mathrm{M}}}}{\partial t}+\nabla \cdot\left(c_{\mathrm{p}} \mathbf{V}_{\mathrm{p}}\right)=0, \quad c_{\mathrm{m}}=\theta * c_{\mathrm{p}}$,

where

$\left\{\begin{array}{l}\phi_{\mathrm{m}} \frac{\partial \theta}{\partial t}-\nabla \cdot\left(\tilde{D}_{\mathrm{m}} \nabla \theta\right) \quad \text { in } \Omega_{\mathrm{M}}, \\ \theta(\boldsymbol{y}, t)=\delta(t) \quad \text { on } \Gamma_{\mathrm{Mp}}, \\ \theta: \Omega_{\mathrm{Mp}} \text {-periodic. }\end{array}\right.$

This homogenised model describes memory effects during solute transport in dual-porosity media with a very tortuous micro-porous matrix.

\subsubsection{Concluding remarks}

The above results show that in contrast with transient fluid flow in dual-porosity media for which memory effects are due to the contrast in permeabilities, these effects may occur during solute transport while there is no such contrast between the transport parameters. This is due to the fact that solute transport by diffusion-convection is a phenomenon with two characteristic times. Consequently, equality of the relevant characteristic times can happen while there is no contrast in diffusivities, but when the local characteristic time of diffusion in the micro-pores reaches the order of the macroscopic characteristic time of convection in the meso-pores. Therefore, memory effects happen for a high Péclet number for which no equivalent continuous macroscopic model can be derived (case ii). A homogenised model with memory effects can be derived in the particular case of a very tortuous microposity which leads to a low contrast in transport properties (case iii), but this situation doesn't seem to fit with those reported in the literature. This analysis explains why, while (as in case i) some authors pretend that in some cases solute transport in dual-porosity media is represented by an equivalent single-porosity model, i.e. by a model with reservoir effect (Berkowitz et al. 1988; Royer et al. 2002), while many in situ and laboratory experimental works report that abnormal concentration profiles are obtained which characterise memory effects and suggest that these effects may systematically occur in a dual-porosity medium (Grisak and Pickens 1980; Rao et al. 1980; Lafolie et al. 1997; Drazer et al. 1998).

\section{Conclusions}

The main result of this work is that a transient phenomenon in a dual-porosity is characterised by:

- a behaviour with reservoir effect when $t_{\mathrm{M}_{l \mathrm{p}}} \ll t_{\mathrm{p}_{\mathrm{L}}}$

The medium behaves like an equivalent single-porosity medium

- a dual-porosity behaviour when $t_{\mathrm{M}_{\mathrm{p}}} \approx t_{\mathrm{p}_{\mathrm{L}}}$

There are coupling and memory effects at the macroscopic scale

- a single-porosity behaviour when $t_{\mathrm{M}_{\mathrm{p}}} \gg t_{\mathrm{p}_{\mathrm{L}}}$

The phenomenon occurs only in the macropores 
where $t_{\mathrm{M} l_{\mathrm{p}}}$ is the local characteristic time in the micro-porous matrix and where $t_{\mathrm{pL}}$ represents the macroscopic characteristic time of the phenomenon. A behaviour with reservoir effect means that a local steady-state regime has been reached in the porous matrix at the time of observation $t_{\mathrm{p}}$. Consequently, there is no memory effects, but the total porosity of the medium is accounted for. Furthermore, two behaviours with reservoir effect are distinguished depending on whether the macroscopic transport effective properties are defined over the whole dual-porosity (global properties) or only over the macro-pore domain (partial properties). A dual-porosity macroscopic behaviour is observed at $t_{\mathrm{p}_{\mathrm{L}}}$ if the phenomenon simultaneously locally occurs in the porous matrix. A single-porosity behaviour corresponds to the case where, at $t_{\mathrm{p}}$, the local phenomenon in the porous matrix has not started yet. The matrix is therefore seen as a non-porous matrix. An important feature of this correlation between the magnitude of the characteristic time ratio and the macroscopic behaviour is that it conditions the apparent behaviour, whatever there exists an homogenised model or not. Furthermore, since the macroscopic behaviour is directly linked to the mechanisms which are involved at the macro-pore scale and more specifically over the micro-porous matrix/meso-pores interface, the above result remains valid in the case of a triple porosity (or of any multiple porosity) medium: the existence of one (or more) additional microscopic scale(s) may have an impact on the value of $t_{\mathrm{M}_{l p}}$, but the correlation between the order of magnitude of $t_{\mathrm{M}_{\mathrm{p}}} / t_{\mathrm{p}_{\mathrm{L}}}$ and the macroscopic behaviour remains the same.

The three types of the above mentioned behaviours may be obtained in the case of compressible fluid flow in dual-porosity media. Another important result of the work is the determination of the rules which govern the order of magnitude of the local velocity ratio (24) for transient fluid flow in dual-porosity media, and which is of crucial importance for homogenising more complex flows (Chastanet et al. 2007).

While dual-porosity effects are directly linked to the permeability contrast for transient fluid flow, memory effects may occur during dispersive solute transport when there is no contrast between transport properties. For a purely diffusive transport in the micro-porosity and a dispersive transport in the mesoporosity, this happens when the local characteristic time of diffusion in the matrix reaches the magnitude of the macroscopic characteristic time of convection. This gives rise to a high Péclet number for which no equivalent continuous macroscopic model can be derived. Memory effects also occur in the particular case of a very tortuous microposity which leads to a contrast in transport properties and for which homogenised models can be derived, but this situation doesn't seem to fit with those reported in the literature. The analysis also explains why, some authors find that in some cases solute transport in dual-porosity media is represented by an equivalent single-porosity model, i.e. by a model with reservoir effect, while many in situ and laboratory experimental works report that abnormal concentration profiles are obtained which characterise memory effects.

Finally, it should be noted that the time analysis being presented in this article should make easier the investigation and interpretation of any novel and more complex phenomenon in dual-porosity media. Besides the results, this paper provides the guidelines on how interpretating the domains of validity of homogenised models. This procedure, which is based on the analysis of the continuous passages between the distinct models, applies to any other problem for which a given microscopic description leads to several macroscopic models.

\section{References}

Arbogast T.: Analysis of the simulation of single-phase flow through a naturally fractured reservoir, SIAM $J$. Numer. Anal, 26, 12-29 (1989). 
Arbogast T.: Computational aspects of dual-porosity models, in Homogenization and Porous Media, U. Hornung Ed., Springer, New-york, (1997).

Arbogast T., Douglas J., Hornung U: Derivation of the double porosity model of single phase flow via homogenization theory, SIAM J. Anal. Math. Anal., 21(4), 823-836, (1990).

Auriault J.-L.: Effective macroscopic description for heat conduction in periodic composites, Int. J. Heat Mass Transfer, 26(6):861-869, (1983).

Auriault J.-L.: Heterogeneous medium. Is an equivalent macroscopic description possible? Int. J. Engng. Sci., 29(7), 785-795, (1991).

Auriault J.-L., Adler P.: Taylor Dispersion in porous media: analysis by multiple scale expansions, Advances in Water Resources, 18, 217-226 (1995).

Auriault J.-L., Boutin C.: Deformable porous media with double porosity. Quasi-statics. I: Coupling effects. Transport in Porous Media, 7, 63-82, (1992).

Auriault J.-L., Boutin C.: Deformable porous media with double porosity. Quasi-statics. II: Memory effects. Transport in Porous Media, 10, 153-169, (1993).

Auriault J.-L., Lewandowska J.: Non-gaussian diffusion modeling in composite porous media by homogenization: tail effect. Transport in Porous Media, 21, 47-70 (1995).

Auriault J.-L., Royer P.: Double conductivity media: a comparison between phenomenological and homogenization approaches, Int. J. Heat Mass Transfer, 36(10), 2613-2621 (1993).

Auriault J.-L., Sanchez-Palencia E., Etude du comportement macroscopique d'un milieu poreux saturé déformable, Journal de Mécanique,16, 37-53, (1977).

Bai M., Elsworth D., Roegiers J.-C: Modeling of naturally fractured reservoirs using deformation dependent fow mechanism, Int. J. Rock Mech. Min. Sci. Geomech. Abstr., 30, 1185-1191 (1993).

Barenblatt G.I., Zheltov Iu, Kochina I.N.: Basic concept in the theory of seepage of homogeneous liquids in fissured rocks, Prikl. Mat. Mekh., 24(5), 852-864 (in Russian); J. Appl. Math. Mech., 24, 1286-1303 (1960).

Barenblatt G.I.: On certain boundary-value-problems for the equations of seepage of liquid in fissured rocks, PMM, 27(2), 348-350, (1963).

Bensoussan A., Lions J.-L. Papanicolaou G.: Asymptotic Analysis for Periodic Structures, North-Holland Publishing Company, Amsterdam, (1978).

Berkowitz B., Bear B., Braester C.: Continuum models for contaminant transport in fractured formations, Water Resour. Res., 24(8), 1225-1236 (1988).

Berryman J.G., Wang F.W.: The elastic coefficients of double-porosity models for fluid transport in jointed rock, Journal of Geophysical Research, 100(B12), 24,611-24-627 (1995).

Beskos D.E., Aifantis E.C.: On the theory of consolidation with double porosity, II, Int. J. Engng. Sci., 24, 1697-1716, (1986).

Bibby R.: Mass transport of solutes in dual-porosity media, Water Resources Research, 24(8), 1225-1236 (1981).

Boutin C. and Auriault J.-C.: Dynamic behaviour of porous media saturated by a viscoelastic fluid. Appliaction to bituminous concretes, International Journal of Engineering Science, 11, 1157-1181 (1990).

Boutin C., Royer P., Auriault J.-L.:Acoustic Absorption of Porous Surfacing with Dual Porosity, International Journal of Solids and Structures, 35, 4709-4737 (1998).

Chastanet J., Royer P., Auriault J.-L.: Flow of low pressure gas through dual-porosity media, Transport in Porous Media, 66, 457-479 (2007)

Coats K., Smith B.: Dead-end pore volume and dispersion in porous media, Society of Petroleum Engineers Journal, III, 73-84 (1964).

Drazer G., Chertcoff R., Bruno L., Rosen M.: Tracer dispersion in double porosity porous media with nonlinear adsorption, Physica A, 257, 371-375.

Elsworrth D, Bai M: Flow-deformation response of dual-porosity media, ASCE J. Geotech. Eng., 118, 107124 (1992)

Grisack G., Pickens J.: Solute transport through fractured media A. The effect of matrix diffusion, Water resour. Res., 16(4), 719-730 (1980).

Hornung U., Showalter R.R.: Diffusion models for fractured media, J. Math. Anal. Applics, 147, 69-80, (1990).

Huyakorn P., Lester B., Mercer J.: An efficient finite element technique for modelling transport in fractured porous media, 1, Single species, Water resources Research, 19(3), 841-854 (1983).

Khaled M.Y., Beskos D. E., Aifantis E.C.: On the theory of consolidation with double porosity-III A finite element formulation, International Journal for Numerical and Analytical Methods in Geomechanics, $\mathbf{8}$, 101-123 (1984).

Lafolie F., Hayot C., Schweich D.: Experiments on solute transport in aggregated porous media: are diffusion within aggregates and hydrodynamic dispersion independent?, Transport in Porous Media, 29, 281-307 
(1997).

Rao P., Rolston D., Jessup R., Davidson J.: Solute transport in aggregated porous media: theoretical and experimental evalutaion, Soil Sci. Soc. Am. Journal, 44, 1139-1146.

Royer P., Auriault J.-L.: Transient quasi-static gas flow through a rigid porous medium with double porosity. Transp. Porous Media 17, 33-57, (1994).

Royer P., Auriault J.-L and C. Boutin: Macroscopic Modeling of Double-Porosity Reservoirs, Journal of Petroleum Science and Engineering 16, 187-202, (1996).

Royer P., Auriault J.-L., Lewandowska J., Serres C...Continuum modelling of contaminant transport in fractured porous media, Transp. Porous Media 49:333-359, (2002).

Royer P., Boutin C.: Time analysis of the thre characteristic behaviours of dual-porosity medie. II: consolidation. Transport in Porous Media, to be submitted.

Sanchez-Palencia, E.: Non-Homogeneous Media and Vibration Theory, Lecture Notes in Physics 127, Springer-Verlag, Berlin, (1980).

Tejchman M.: Solute transport through single and dual porosity media. Mathematical modelling by homogenization and column experimental investigations, PhD Thesis, Université Joseph Fourier, Grenoble, France.

Warren J.E, Root P.J.: The behavior of naturally fractured reservoirs, J. Soc. Petroleum Engineers, 3, 245-255 (1963).

Wilson R.K., Aifantis E.C.: On the theory of consolidation with double porosity, Int. J. Engng. Sci, 20(9), 1009-1035 (1982). 